\title{
On PIR and Symmetric PIR from Colluding Databases with Adversaries and Eavesdroppers
}

\author{
Qiwen Wang and Mikael Skoglund
}

\begin{abstract}
We consider the problem of private information retrieval (PIR) and symmetric private information retrieval (SPIR) from replicated databases with colluding servers, in the presence of Byzantine adversaries and eavesdroppers. Specifically, there are $K$ messages replicatively stored at $N$ databases. A user wants to retrieve one message by communicating with the databases, without revealing the identity of the message retrieved. For $T$-colluding databases, any $T$ out of $N$ databases may communicate their interactions with the user to guess the identity of the requested message. We consider the situation where the communication system can be vulnerable to attachers, namely, there is an adversary in the system that can tap in on or even try to corrupt the communication. The capacity is defined as the maximum number of information bits of the desired message retrieved per downloaded bit. For SPIR, it is further required that the user learns nothing about the other $K-1$ messages in the database. Three types of adversaries are considered: a Byzantine adversary who can overwrite the transmission of any $B$ servers to the user; a passive eavesdropper who can tap in on the incoming and outgoing transmissions of any $E$ servers; and a combination of both - an adversary who can tap in on a set of any $E$ nodes, and overwrite the transmission of a set of any $B$ nodes. The problems of SPIR with colluding servers and the three types of adversaries are named T-BSPIR, T-ESPIR and T-BESPIR respectively. We derive the capacities of the three secure SPIR problems. The results resemble those of secure network coding problems with adversaries and eavesdroppers. The capacity of $T$-colluding PIR with Byzantine adversaries is characterized in [1]. In this work, we consider $T$-colluding PIR with an eavedropper (named T-EPIR). We derive the T-EPIR capacity when $E \geq T$; for the case where $E \leq T$, we find an outer bound (converse bound) and an inner bound (achievability) on the optimal achievable rate.
\end{abstract}

\section{INTRODUCTION}

With the evolution of the Internet of things, big data, cloud storage and computing, much of the emerging technology relies on open systems and networks, which brings up new challenges in preserving data privacy and security in such applications. Recently, various privacy and security problems in the storage, fetching, and communication of data have been studied using tools from information theory, among which the problem of private information retrieval brought up in the $90 \mathrm{~s}$

Manuscript received December 22, 2017; revised August 15, 2018; accepted October 08, 2018. This paper was presented in part at the 55th Annual Allerton Conference on Communication, Control, and Computing, Oct 2017.

This work was supported by the Knut and Alice Wallenberg Foundation.

Q. Wang is with the Department of Information Science and Engineering, KTH Royal Institute of Technology. (email: qiwenw@kth.se).

M. Skoglund is with the Department of Information Science and Engineering, KTH Royal Institute of Technology. (email: skoglund@ee.kth.se).

Copyright (c) 2017 IEEE. Personal use of this material is permitted. However, permission to use this material for any other purposes must be obtained from the IEEE by sending a request to pubs-permissions@ieee.org. in the computer science community has drawn new attention from researchers working in information theory.

Consider the situation where a user wants to retrieve a file (message) from a remotely stored database, the nature of the data might be privacy-sensitive, for example medical records, stock prices etc., such that the user does not want to reveal the identity of the data retrieved. This is known as the problem of private information retrieval (PIR). In some cases, the privacy of the database needs also to be preserved. For example, if a user wants to retrieve his/her medical data from a database, it is hoped that the user obtains no information about other users' medical records. This is known as the problem of symmetric private information retrieval (SPIR).

The problem of PIR and SPIR was firstly studied in the computer science literature. In [2], [3], it is shown that if the messages are stored at a single database, the only possible scheme for the user is to download all the messages to guarantee information-theoretic privacy, which is inefficient in practice. It is further shown that the communication cost can be reduced in sublinear scale by replicating the database at multiple non-colluding servers [3]. To further protect the privacy of the database such that the user obtains no more information regarding the other messages besides the requested message, the problem of SPIR is introduced [4]. In [2]-[4], the collection of messages stored at each database is modeled as a bit string, and the user wishes to retrieve a single bit. In these works, the communication cost is measured as the sum of the transmission at the querying phase from user to servers and at the downloading phase from servers to user.

When the message size is significantly large and the target is to minimize the communication cost of only the downloading phase, the metric of the downloading cost is defined as the number of bits downloaded per bit of the retrieved message, and the reciprocal of which is named the retrieval rate. The supremum of all achievable retrieval rates is defined as the PIR capacity. A series of recent works derive information-theoretic limits of various versions of the PIR problem [1], [5]-[10] etc. The leading work in the area is by Sun and Jafar [5], where the authors characterize the capacity of the PIR problem with replicated databases. In subsequent works by Sun and Jafar [6], [7], the PIR capacity with replicated databases and colluding servers, and the SPIR capacity with replicated (noncolluding) databases are derived. In [1], [8], [9], Banawan and Ulukus find the capacity of the PIR problem with coded databases, multi-message PIR with replicated databases, and the PIR problem with colluding and Byzantine databases. In our previous works [10], [11], we derive the capacity of the SPIR problem with coded databases, and linear SPIR with 
colluding and coded databases.

Another series of works focus more on the coding structure of the storage system, and study schemes and information limits for various PIR problems with coded databases [12] [16]. In [12], PIR is achieved by downloading one extra bit other than the desired file, given that the number of storage nodes grows with file size, which can be impractical in some storage systems. In [13], storage overhead can be reduced by increasing the number of storage nodes. In [14], tradeoff between storage cost and downloading cost is analyzed. Subsequently in [15], explicit schemes which match the tradeoff in [14] are presented. It is worth noting that in [8], the capacity of PIR for coded databases is settled, which improves the results in [14], [15]. In [16], the authors present a framework for PIR from coded databases with colluding servers.

In this work, we consider the PIR and SPIR problems from replicated and colluding databases, in the presence of Byzantine adversaries and eavesdroppers. Specifically, there are $K$ independent messages, which are replicated at $N$ databases. A user wants to retrieve one of the $K$ messages with zero probability of error, without revealing the message index to any database (server). For SPIR, it is further required that the user does not learn any information about the other $K-1$ messages from the downloads. Any $T$ out of the $N$ databases may collude, that is, they may share their communication with the user to infer the identity of the requested file. The communication in the system is not secure, that is, there is an adversary who can tap in on or even corrupt the transmissions in the system. A Byzantine adversary can overwrite the transmission of any $B$ databases to the user. A passive eavesdropper can tap in on the incoming and outgoing transmissions of any $E$ databases.

The main contribution of this work is the characterization of $T$-colluding SPIR capacity with Byzantine adversaries (TBSPIR), with eavesdroppers (T-ESPIR), and with adversaries who can both eavesdrop and jam the communication (TBESPIR). In analogy to previous results on SPIR [7], [10], in the non-trivial context where the database comprises at least two messages, the servers need to share common randomness which is independent from the messages and meanwhile unavailable to the user. Furthermore, in the case with an eavesdropper who can tap in on a set of the nodes and is curious about the database, the utility of the shared common randomness is two-fold in the sense that it also protects the messages from the eavesdropper. We show that the informationtheoretical capacity of the T-BSPIR problem equals $1-\frac{2 B+T}{N}$, if the servers share common randomness with amount at least $\frac{T}{N-2 B-T}$ times the message size. Otherwise, the capacity equals zero. The information-theoretical capacity of the TESPIR problem is proved to equal $1-\frac{\max (T, E)}{N}$, if the servers share common randomness with amount at least $\frac{\max (T, E)}{N-\max (T, E)}$ times the message size. Finally, we extend to the case where the adversary can both eavesdrop and corrupt communications, and characterize the capacity to be $1-\frac{2 B+\max (T, E)}{N}$, where the common randomness shared by the servers should be at least $\frac{\max (T, E)}{-2 B-\max (T, E)}$ times the message size. The results resemble the corresponding ones for the capacity of secure network coding (NC) with adversaries [17] (summarized in Table I). For zero probability of decoding error, there is a factor 2 in front of the number of jamming links (in NC results) and jamming servers (in SPIR results), i.e., $B$ erroneous answers result in a waste of $2 B$ answers.

For the PIR versions of the problems, the capacity of $T$ colluding PIR with Byzantine adversaries (T-BPIR) was found in [1] to be

$$
C_{\text {T-BPIR }}=\frac{N-2 B}{N} \cdot \frac{1-\frac{T}{N-2 B}}{1-\left(\frac{T}{N-2 B}\right)^{K}} .
$$

For TPIR with eavesdroppers (T-EPIR), to prevent the eavesdropper from learning the messages, the databases share some common randomness. We find that when $E \geq T$, the T-EPIR capacity equals $1-\frac{E}{N}$ when the ratio of the amount of common randomness to the message size is at least $\frac{E}{N-E}$. For the case where $E<T$, We derive an outer bound (converse bound) on the optimal achievable rate that $R^{*} \leq\left(1-\frac{T}{N}\right) \frac{1-\frac{E}{N} \cdot\left(\frac{T}{N}\right)^{K-1}}{1-\left(\frac{T}{N}\right)^{K}}$. We also obtain a lower bound (converse bound) on the ratio of the amount of common randomness to the message size that $\rho^{*} \geq \frac{\frac{E}{N}\left(1-\left(\frac{T}{N}\right)^{K}\right)}{\left(1-\frac{T}{N}\right)\left(1-\frac{E}{N} \cdot\left(\frac{T}{N}\right)^{K-1}\right)}$ to achieve positive rates. For the achievability, we propose a scheme which achieves the rate (inner bound) $R=\frac{1-\frac{T}{N}}{1-\left(\frac{T}{N}\right)^{K}}-\frac{E}{K N}$. The amount of shared common randomness used in the achievable scheme is $\frac{\frac{E}{N}\left(1-\left(\frac{T}{N}\right)^{K}\right)}{1-\frac{T}{N}-\frac{E}{K N}\left(1-\left(\frac{T}{N}\right)^{K}\right)}$ times the file size. ${ }^{1}$ For illustration, we plot the results in Figure 1 and Figure 2 for some chosen parameters. It can be observed from the figures that the gap between inner and outer bounds decays and vanishes as $K$ tends to infinity. Table II summarizes the results on secure PIR and SPIR.

\section{MODEL}

\section{A. Notation}

Let $[m: n]$ denote the set $\{m, m+1, \ldots, n\}$ for $m \leq n$. To simplify the notation, denote the set of random variables $\left\{X_{m}, X_{m+1}, \ldots, X_{n}\right\}$ by $X_{[m: n]}$. For an index set $\mathcal{I}=$ $\left\{i_{1}, i_{2}, \ldots, i_{n}\right\}$, denote the set of variables with the index set $\left\{X_{i}: i \in \mathcal{I}\right\}$ by $X_{\mathcal{I}}$. For a matrix $\mathbf{S}$, let $\mathbf{S}[:, \mathcal{I}]$ denote the submatrix of $\mathbf{S}$ comprised of the columns corresponding to the index set $\mathcal{I}$. The transpose of matrix $\mathbf{G}$ is denoted by $\mathbf{G}^{\mathrm{T}}$. Let $\sim$ denote the statistical equivalence between random variables, that is, if $X \sim Y$, then $X$ and $Y$ are identically distributed.

\section{B. Problem Description}

Replicated databases: A collection of $K$ independent messages (files), denoted by $W_{1}, \ldots, W_{K}$, are replicatively stored at $N$ databases (also named servers or nodes). Each message consists $L$ information bits. Therefore, for any $k \in[1: K]$,

$$
\begin{aligned}
& H\left(W_{k}\right)=L ; H\left(W_{1}, \ldots, W_{K}\right)=K L . \\
& { }^{1} \text { In our recent work [18], we characterize the T-EPIR } \\
& \text { capacity when } E<T \text { to be } C_{\text {T-EPIR }}=\left(1-\frac{E}{N}\right) . \\
& \left(1+\frac{T-E}{N-E}+\left(\frac{T-E}{N-E}\right)^{2}+\cdots+\left(\frac{T-E}{N-E}\right)^{K-1}\right)^{-1} .
\end{aligned}
$$




\begin{tabular}{|c|c|c|}
\hline Adversary & Secure network coding [17] & TSPIR \\
\hline \hline Byzantine & $C-2 Z_{O}($ Corollary 4.1) & $C_{\text {T-BSPIR }}=1-\frac{2 B+T}{N}$ \\
\hline Eavesdropping & $C-Z_{I}$ (Theorem 3.1) & $C_{\text {T-ESPIR }}=1-\frac{\max (T, E)}{N}$ \\
\hline Byzantine and eavesdropping & $C-2 Z_{O}-Z_{I}$ (Theorem 5.1) & $C_{\text {T-BESPIR }}=1-\frac{2 B+\max (T, E)}{N}$ \\
\hline
\end{tabular}

TABLE I: Comparison between results on secure network coding (where the min-cut is $C$; the adversary can eavesdrop on $Z_{I}$ links and jam $Z_{O}$ links) and secure SPIR. Here, zero probability of decoding error is required.

\begin{tabular}{|c|c|c|}
\hline Adversary & TPIR & TSPIR \\
\hline \hline Byzantine & $C_{\text {T-BPIR }}=\frac{N-2 B}{N} \cdot \frac{1-\frac{T}{N-2 B}}{1-\left(\frac{T}{N-2 B}\right)^{K}}[1]$ & $C_{\text {T-BSPIR }}=1-\frac{2 B+T}{N}$ \\
\hline \multirow{2}{*}{ Eavesdropping } & When $E \geq T, C_{\text {T-EPIR }}=1-\frac{E}{N} ;$ & \\
& $\begin{array}{l}\text { When } E<T, \\
1-\frac{T}{N}\end{array}-\frac{E}{K N} \leq R^{*} \leq\left(1-\frac{T}{N}\right) \frac{1-\frac{E}{N} \cdot\left(\frac{T}{N}\right)^{K-1}}{1-\left(\frac{T}{N}\right)^{K}}$ & $C_{\text {T-ESPIR }}=1-\frac{\max (T, E)}{N}$ \\
\hline Byzantine and eavesdropping & open & $C_{\text {T-BESPIR }}=1-\frac{2 B+\max (T, E)}{N}$ \\
\hline
\end{tabular}

TABLE II: Results on secure PIR and SPIR.

User queries: A user wants to retrieve a message $W_{\kappa}$ with index $\kappa$ from the database, where the desired message index $\kappa$ is chosen uniformly from $[1: K]$. Let $\mathcal{U}$ denote a random variable privately generated by the user, which represents the randomness of the query scheme followed by the user. The random variable $\mathcal{U}$ is generated independently of the messages and the desired message index. Let the realization of the message index $\kappa$ be $k$, based on the realization of the desired message index $k$ and the realization of $\mathcal{U}$, the user generates and sends queries to all nodes, where the query received by node- $n$ is denoted by $Q_{n}^{[k]}$. Let $\mathcal{Q}=\left[Q_{n}^{[k]}\right]_{n \in[1: N], k \in[1: K]}$ denote the complete query scheme, namely, the collection of all queries under all cases of desired message index. We have that $H(\mathcal{Q} \mid \mathcal{U})=0$.

Common randomness: Let random variable $S$ denote the common randomness shared by all databases, the realization of which is known to all the databases but unavailable to the user and the eavesdropper. For the T-EPIR problem, the common randomness is utilized to protect the systemprivacy (5) below, that is, to prevent the eavesdropper from learning the messages. For the SPIR problems, the purpose of the common randomness is to protect database-privacy (4) from the user. Therefore, in the scenario with eavesdroppers and SPIR is required, the utility of the common randomness is twofold.

Database answers: The databases generate answers according to the agreed scheme with the user based on the received query $Q_{n}^{[k]}$, the stored messages $W_{[1: K]}$, and the common randomness $S$. The answer generated and sent to the user by node $n$ is denoted by $A_{n}^{[k]}$.

Eavesdropper: A passive eavesdropper can tap in on the incoming and outgoing transmissions of $E$ nodes in the system. The eavesdropper is "nice but curious," in the sense that the goal of the eavesdropper is to obtain some information about the database, without corrupting any transmission. The user has no knowledge of the identity of the nodes tapped on by the eavesdropper.

Adversary: A Byzantine adversary can overwrite the answers of a set $\mathcal{B}$ of at most $B$ nodes, called corrupted nodes, pretending to send answers to the user from the corrupted nodes to confuse the user. The nodes that are not corrupted by the adversary are called authentic nodes. The user has no knowledge of the identity of the corrupted nodes, other than knowing that at most $B$ nodes are affected. The answers overwritten and sent to the user are denoted by $\tilde{A}_{\mathcal{B}}^{[k]}$. We assume the Byzantine adversary is omniscient, that is, the adversary can observe all transmissions and corrupt the $B$ answers in a worst-case way that confuses the user the most. ${ }^{2}$ The model studied in [1] considers the servers to be adversarial, hence is different from our model. In [1], there are $B$ Byzantine adversarial nodes who send arbitrary or worst-case answers to the user, hence have limited observations (each server observes the queries/answers by its own and the servers colluding with it). ${ }^{3}$

We also investigate the case in which an adversary is a combination of the above two types. The adversary can tap in on the incoming and outgoing communications of any set $\mathcal{E}$ with $E$ nodes, and can overwrite the answers of any set $\mathcal{B}$ with $B$ nodes. The two sets can be overlapping or disjoint. In this case, the observation of the adversary is the communications of the servers $\mathcal{E}$, hence the adversary is not omniscient.

PIR and SPIR: Based on the received answers $A_{[1: N]}^{[k]}$ (for

\footnotetext{
${ }^{2}$ In this scenario, the adversary observes all communications. There is no requirement on the privacy of the user's desired message index or the privacy of the messages to the adversary.

${ }^{3}$ For zero-error decodability, the knowledge of the adversary does not affect the result, because the adversary could "happen" to generate the worst-case corrupted answers without knowing the transmissions in the system, in which case the communication scheme should still prevent the user from decoding the desired message wrong. We allow the omniscience of the adversary to emphasize that the result holds for the strongest possible adversary.
} 
the case with Byzantine adversary, we abuse the notation and let $\left.A_{[1: N]}^{[k]}=\left\{A_{[1: N] \backslash \mathcal{B}}^{[k]}, \tilde{A}_{\mathcal{B}}^{[k]}\right\}\right)$ and the query scheme $\mathcal{Q}$, the user shall be able to decode the requested message $W_{k}$ with zero error, i.e.,

$$
\text { Decodability: } \quad H\left(W_{k} \mid A_{[1: N]}^{[k]}, \mathcal{Q}\right)=0 .
$$

Any set of $T$ databases may collude to guess the requested message index, by communicating their interactions with the user. The user-privacy constraint below should be satisfied for all variants of PIR problems.

- User-privacy: any $T$ colluding databases shall not be able to obtain any information regarding the identity of the requested message, i.e.,

$$
I\left(\kappa ; Q_{\mathcal{T}}^{[\kappa]}, A_{\mathcal{T}}^{[\kappa]}, W_{[1: K]}, S\right)=0, \forall \mathcal{T} \subset[1: N],|\mathcal{T}|=T
$$

For SPIR problems, the database-privacy constraint below should be also satiesfied.

- Database-privacy: the user shall learn no information regarding other messages in the database, that is, defining $W_{\bar{\kappa}}=\left\{W_{1}, \ldots, W_{\kappa-1}, W_{\kappa+1}, \ldots, W_{K}\right\}$,

$$
I\left(W_{\bar{\kappa}} ; A_{[1: N]}^{[\kappa]}, \mathcal{Q}, \kappa\right)=0 .
$$

For the scenario with passive eavesdropper (T-EPIR and T-ESPIR) and the case with the combination adversary (TBESPIR), one more privacy constraint must be satisfied to protect the database from the eavesdropper.

- System-privacy (security): For any set of databases $\mathcal{E}$ with size at most $E$, and for any $k \in[1: K]:^{4}$

$$
I\left(W_{[1: K]} ; Q_{\mathcal{E}}^{[k]}, A_{\mathcal{E}}^{[k]}\right)=0 .
$$

We use the same definition as in [10] for the rate and capacity of PIR and SPIR schemes.

Definition 1. The rate of a PIR (SPIR) scheme is the number of information bits of the requested message retrieved per downloaded answer bit. By symmetry among all messages, for any $k \in[1: K]$,

$$
R \triangleq \frac{H\left(W_{k}\right)}{\sum_{n=1}^{N} H\left(A_{n}^{[k]}\right)} .
$$

The optimal achievable rate is denoted by $R^{*}$. The capacity $C$ is the supremum of the achievable $R$ over all schemes.

Definition 2. The secrecy rate is the amount of common randomness shared by the storage nodes relative to the message size, that is

$$
\rho_{T-E P I R} \triangleq \frac{H(S)}{H\left(W_{1}\right)} .
$$

\footnotetext{
${ }^{4}$ In this work, we consider the eavesdropper who can tap in on the uplink and downlink of the same set of nodes. If the eavesdropper can tap in on the entire uplink (with system-privacy constraint $I\left(W_{[1: K]} ; Q_{[1: N]}^{[k]}, A_{\mathcal{E}}^{[k]}\right)=$ 0 ), or even knows the whole query scheme (with system-privacy constraint $\left.I\left(W_{[1: K]} ; \mathcal{Q}, A_{\mathcal{E}}^{[k]}\right)=0\right)$, the bounds and capacity results in this work still hold.
}

\section{MAIN RESUlT}

\section{A. T-BSPIR}

When there is only one message in the database, i.e. $K=1$, database-privacy is guaranteed automatically, because there is no other message to protect from the user in the database. Therefore, the T-BSPIR problem reduces to the T-BPIR problem, and from [1], the capacity is $1-\frac{2 B}{N}$ if $N>2 B+T$. In fact, when $K=1$, user-privacy is also trivial, since there is only one message that the user can request for. This is the reason the parameter $T$ does not appear in the capacity result $1-\frac{2 B}{N}$. Therefore, the condition can be relaxed to that if $N \geq 2 B+1$, the capacity of T-BSPIR when $K=1$ is $1-\frac{2 \bar{B}}{N}$. If $N \leq 2 B$, the user cannot successfully retrieve the message regardless of how much is information downloaded, i.e. the capacity is 0 . When $K \geq 2$, T-BSPIR is non-trivial and our main result is summarized below.

Theorem 1. For symmetric private information retrieval from a database with $K \geq 2$ messages which are replicated at $N$ servers, where any $T$ servers may collude and a Byzantine adversary can corrupt the answers of any $B$ servers, if $N>$ $2 B+T$, the capacity is

$$
C_{T-B S P I R}=\left\{\begin{array}{ll}
1-\frac{2 B+T}{N}, & \text { if } \rho_{T-B S P I R} \geq \frac{T}{N-2 B-T} \\
0, & \text { otherwise }
\end{array} .\right.
$$

Remark: In [1], the authors show that the T-BPIR capacity is $\frac{N-2 B}{N} \cdot \frac{1-\frac{T}{N-2 B}}{1-\left(\frac{T}{N-2 B}\right)^{K}}$. It can be observed that as the number of messages $K$ tends to infinity, their T-BPIR capacity approaches to our T-BSPIR capacity. The intuition is that, when the number of messages increases, the penalty in the downloading rate to protect database-privacy decays. When there are asymptotically infinitely many messages, the information rate the user can learn about the database from finite downloaded symbols vanishes.

\section{B. T-ESPIR}

When there is only one message in the database, both database-privacy and user-privacy become trivial. The only privacy constraint needed to be guaranteed is that the eavesdropper learns no information of the database (5). It can be easily checked that the capacity equals $1-\frac{E}{N}$. When $K \geq 2$, the capacity of T-ESPIR is summarized below.

Theorem 2. For symmetric private information retrieval from a database with $K \geq 2$ messages which are replicated at $N$ servers, where any $T$ servers may collude and an eavesdropper can tap in on the communication of any E servers, the capacity is

$$
C_{\text {T-ESPIR }}= \begin{cases}1-\frac{\max (T, E)}{N}, & \text { if } \rho_{T-E S P I R} \geq \frac{\max (T, E)}{N-\max (T, E)} \\ 0, & \text { otherwise }\end{cases}
$$

\section{T-BESPIR}

Theorem 3. For symmetric private information retrieval from a database with $K \geq 2$ messages which are replicated at $N$ 
servers, where any $T$ servers may collude, an adversary who is curious about the messages can tap in on the communication of any $E$ servers and corrupt the answers of any $B$ servers, the capacity is

$C_{T-B E S P I R}=\left\{\begin{array}{ll}1-\frac{2 B+\max (T, E)}{N}, & \text { if } \rho_{T-B E S P I R} \geq \frac{\max (T, E)}{N-2 B-\max (T, E)} \\ 0, & \text { otherwise }\end{array}\right.$.

\section{T-EPIR}

Theorem 4 (Capacity when $E \geq T$ ). For T-EPIR with $K$ messages replicated at $N$ servers, where any $T$ servers may collude and an eavesdropper can tap in on the communication of any $E$ servers, when $E \geq T$, the capacity is

$$
C_{\text {T-EPIR }}=\left\{\begin{array}{ll}
1-\frac{E}{N}, & \text { if } \rho_{\text {T-EPIR }} \geq \frac{E}{N-E} \\
0, & \text { otherwise }
\end{array} .\right.
$$

Remark: It can be observed that when $E \geq T$, the T-EPIR capacity is equal to the T-ESPIR capacity (Theorem 2). It is surprising that in this case, database-privacy can be guaranteed with no extra penalty on the achievable rate.

Theorem 5 (Outer Bound when $E \leq T$ ). For T-EPIR with $K$ messages replicated at $N$ servers, where any $T$ servers may collude and an eavesdropper can tap in on the communication of any $E$ servers, when $E \leq T$,

$$
R_{\text {T-EPIR }}^{*} \leq \bar{R}_{\text {T-EPIR }}=\left(1-\frac{T}{N}\right) \frac{1-\frac{E}{N} \cdot\left(\frac{T}{N}\right)^{K-1}}{1-\left(\frac{T}{N}\right)^{K}} .
$$

The secrecy rate, i.e. the ratio of the amount of common randomness to the message size is at least $\rho_{\text {T-EPIR }} \geq$ $\frac{\frac{E}{N}\left(1-\left(\frac{T}{N}\right)^{K}\right)}{\left(1-\frac{T}{N}\right)\left(1-\frac{E}{N} \cdot\left(\frac{T}{N}\right)^{K-1}\right)}$.

Theorem 6 (Inner Bound when $E \leq T$ ). For T-EPIR with $K$ messages replicated at $N$ servers, where any $T$ servers may collude and an eavesdropper can tap in on the communication of any $E$ servers, when $E \leq T$,

$$
R_{T-E P I R}^{*} \geq \underline{R}_{T-E P I R}=\frac{1-\frac{T}{N}}{1-\left(\frac{T}{N}\right)^{K}}-\frac{E}{K N}
$$

In Figure 1 and Figure 2, the results of Theorems 4- 6 are plotted for several sets of parameters. It can be observed from the figures that when the number of messages $K$ increases, the gap between the inner and outer bounds decays and vanishes as $K \rightarrow \infty$.

\section{T-BSPIR}

\section{A. Achievability}

In this section, we present a general scheme which achieves the maximum T-BSPIR rate $1-\frac{2 B+T}{N}$ when the secrecy rate is $\frac{T}{N-2 B-T}$. The main concepts of the construction are:

- The queries received by any set of $T$ servers are mutually independent, and are independent of the desired message

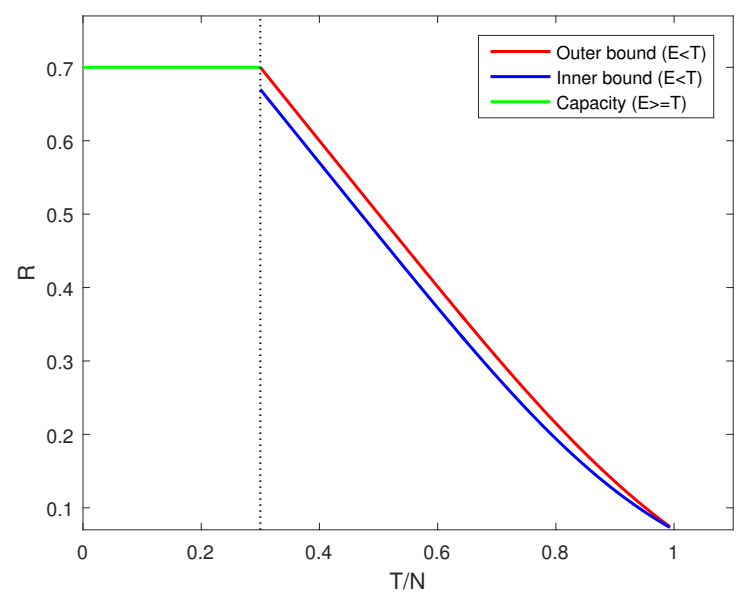

(a) $N=10, E=3, K=10$

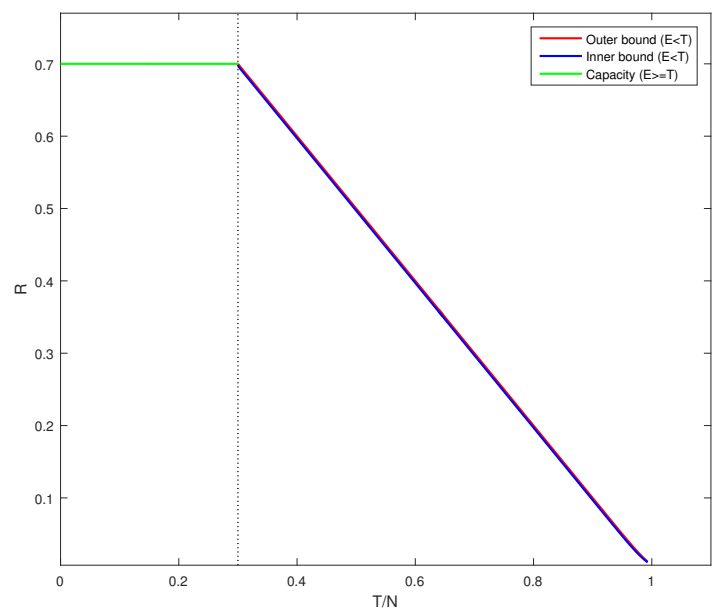

(b) $N=10, E=3, K=100$

Fig. 1: Plot of the inner and outer bounds of T-EPIR as functions of $\frac{T}{N}$.

index $k$. This is achieved by expanding $T$ independent query vectors with an $(N, T)$-MDS code.

- Because the answers received from any $B$ servers might be erroneous, the $N$ answers are formalized in a form of $(N, N-2 B)$-MDS code, such that the user can correct up to $B$ errors.

Assume each message comprises $L=N-2 B-T$ symbols from a large enough field $\mathbb{F}_{q} \cdot{ }^{5}$ Let the vector $\mathbf{W}=$ $\left(w_{1}^{[1]}, \ldots, w_{N-2 B-T}^{[1]}, \ldots, w_{1}^{[K]}, \ldots, w_{N-2 B-T}^{[K]}\right)$ represent the collection of all $K$ messages, which is stored at each server. The user wants to retrieve $W_{k}=\left(w_{1}^{[k]}, \ldots, w_{N-2 B-T}^{[k]}\right)$ privately.

The user generates the queries following the steps below:

Step 1: Generates $T$ independent uniformly random vectors $U_{1}, \ldots, U_{T}$ of length $K(N-2 B-T)$ over $\mathbb{F}_{q}$. Let the $K(N-$ $2 B-T) \times T$ matrix $\mathbf{U}$ denote $\left[U_{1}, \ldots, U_{T}\right]$.

\footnotetext{
${ }^{5}$ The field size should be large enough such that the MDS codes used in
} the construction exist. 


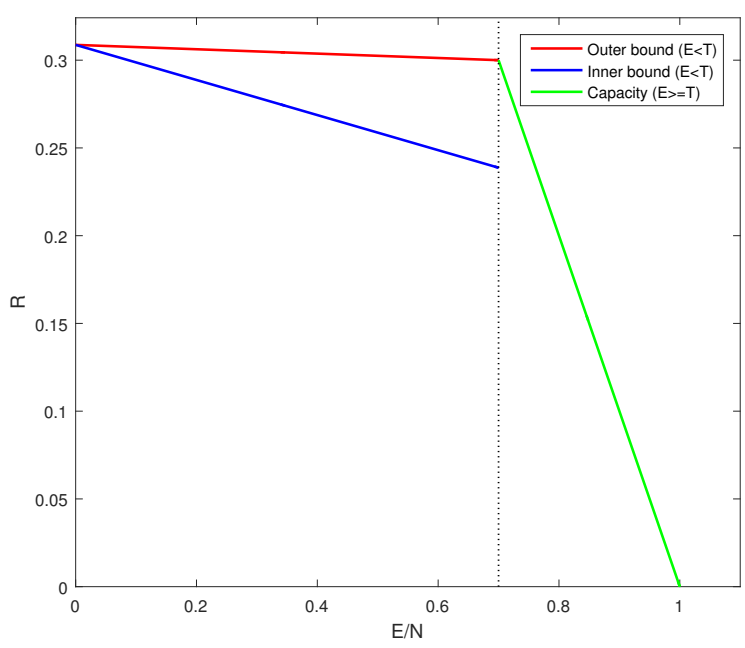

(a) $N=10, T=7, K=10$

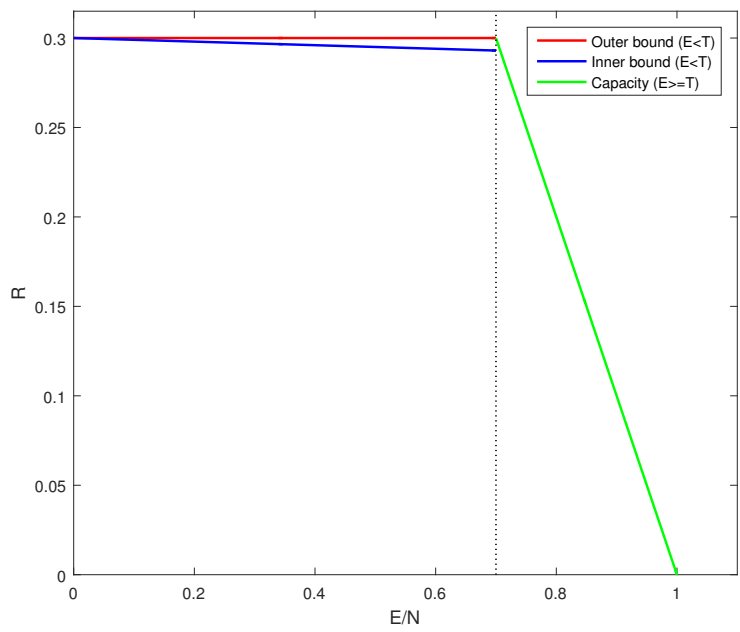

(b) $N=10, T=7, K=100$

Fig. 2: Plot of the inner and outer bounds of T-EPIR as functions of $\frac{E}{N}$.

Step 2: Let $e_{i}^{[k]}$ denote the length- $(K(N-2 B-T))$ unit vector where only the $((k-1)(N-2 B-T)+i)$ th entry is 1 and all the other entries are 0 's. The purpose of $e_{i}^{[k]}$ is to retrieve the $i$ th entry of $W_{k}$. Let the $K(N-2 B-T) \times(N-2 B-T)$ matrix e denote $\left[e_{1}^{[k]}, e_{2}^{[k]}, \ldots, e_{N-2 B-T}^{[k]}\right]$.

Step 3: Let $\left\{\lambda_{1}, \ldots, \lambda_{N}\right\}$ be $N$ distinct nonzero elements from $\mathbb{F}_{q}$. Let $\mathbf{G}_{\mathbf{U}}$ be the generating matrix of an $(N, T)$ generalized-Reed-Solomon (GRS) code with code locators $\left\{\lambda_{1}, \ldots, \lambda_{N}\right\}$ and column multipliers all be 1 . That is,

$$
\mathbf{G}_{\mathbf{U}}=\left[\begin{array}{cccc}
1 & 1 & \ldots & 1 \\
\lambda_{1} & \lambda_{2} & \ldots & \lambda_{N} \\
\vdots & \vdots & \ddots & \vdots \\
\lambda_{1}^{T-1} & \lambda_{2}^{T-1} & \ldots & \lambda_{N}^{T-1}
\end{array}\right]
$$

Let $\mathbf{G}_{\mathbf{e}}$ be the generating matrix of an $(N, N-2 B-T)$-GRS code with code locators $\left\{\lambda_{1}, \ldots, \lambda_{N}\right\}$ and column multipliers

$$
\begin{aligned}
& \left\{\lambda_{1}^{T-1}, \ldots, \lambda_{N}^{T-1}\right\} \text {. That is, } \\
& \begin{aligned}
\mathbf{G}_{\mathbf{e}}= & {\left[\begin{array}{cccc}
1 & 1 & \ldots & 1 \\
\lambda_{1} & \lambda_{2} & \ldots & \lambda_{N} \\
\vdots & \vdots & \ddots & \vdots \\
\lambda_{1}^{N-2 B-T-1} & \lambda_{2}^{N-2 B-T-1} & \ldots & \lambda_{N}^{N-2 B-T-1}
\end{array}\right] } \\
& \cdot \operatorname{diag}\left(\lambda_{1}^{T}, \lambda_{2}^{T}, \ldots, \lambda_{N}^{T}\right) \\
= & {\left[\begin{array}{cccc}
\lambda_{1}^{T} & \lambda_{2}^{T} & \ldots & \lambda_{N}^{T} \\
\lambda_{1}^{T+1} & \lambda_{2}^{T+1} & \ldots & \lambda_{N}^{T+1} \\
\vdots & \vdots & \ddots & \vdots \\
\lambda_{1}^{N-2 B-1} & \lambda_{2}^{N-2 B-1} & \ldots & \lambda_{N}^{N-2 B-1}
\end{array}\right] }
\end{aligned}
\end{aligned}
$$

Step 4: Generate the $N$ query vectors $Q_{1}^{[k]}, \ldots, Q_{n}^{[k]}$ by

$$
\begin{aligned}
{\left[Q_{1}^{[k]}, \ldots, Q_{n}^{[k]}\right] } & =\mathbf{U G}_{\mathbf{U}}+\mathbf{e G}_{\mathbf{e}} \\
& =[\mathbf{U}, \mathbf{e}] \cdot\left[\begin{array}{l}
\mathbf{G}_{\mathbf{U}} \\
\mathbf{G}_{\mathbf{e}}
\end{array}\right]
\end{aligned}
$$

The user sends the query vectors generated from equation (12) to the servers.

All the servers share $T$ symbols $S_{1}, \ldots, S_{T}$ that are uniformly and independently chosen from $\mathbb{F}_{q}$, which are unavailable to the user. The servers generate their answers by taking the inner product of the query vectors they receive and the stored data vector $\mathbf{W}$, then add on a linear combination of $S_{1}, \ldots, S_{T}$. Specifically,

$$
A_{n}^{[k]}=\left\langle Q_{n}^{[k]}, \mathbf{W}\right\rangle+\sum_{j=1}^{T} \lambda_{n}^{j-1} S_{j} .
$$

There are at most $B$ servers corrupted by the Byzantine adversary, who generate arbitrary (or even malicious) answers $\tilde{A}_{n}^{[k]}$ to confuse the user. Assume the Byzantine adversary generates answers of the same size as the authentic servers, i.e. the size the user expects to receive. This assumption can be made without loss of generality because there is no motivation for the adversary to change the answer sizes. Otherwise, the user can easily identify the corrupted answers and treat them as erasures.

To see that the user can decode $W_{k}$ successfully, firstly we look at the $N$ correct answers. Denote $X_{j}=\left\langle U_{j}, \mathbf{W}\right\rangle+S_{j}$, where $j=1, \ldots, T$. From (12) and (13), $A_{n}^{[k]}=X_{1}+\lambda_{n} X_{2}+$ $\cdots+\lambda_{n}^{T-1} X_{T}+\lambda_{n}^{T} w_{1}^{[k]}+\cdots+\lambda_{n}^{N-2 B-1} w_{N-2 B-T}^{[k]}$. Hence,

$$
\left[A_{1}^{[k]}, \ldots, A_{n}^{[k]}\right]=\left[X_{1}, \ldots, X_{T}, w_{1}^{[k]}, \ldots, w_{N-2 B-T}^{[k]}\right] \cdot \mathbf{G},
$$

where

$$
\begin{aligned}
\mathbf{G} & =\left[\mathbf{G}_{\mathbf{U}}, \mathbf{G}_{\mathbf{e}}\right]^{\mathrm{T}} \\
& =\left[\begin{array}{cccc}
1 & 1 & \ldots & 1 \\
\lambda_{1} & \lambda_{2} & \ldots & \lambda_{N} \\
\vdots & \vdots & \ddots & \vdots \\
\lambda_{1}^{N-2 B-1} & \lambda_{2}^{N-2 B-1} & \ldots & \lambda_{N}^{N-2 B-1}
\end{array}\right] .
\end{aligned}
$$

It can be observed that $\mathbf{G}$ is the generating matrix of an $(N, N-2 B)$-GRS code with code locators $\left\{\lambda_{1}, \ldots, \lambda_{N}\right\}$ and column multipliers all equal 1 . Therefore, when at most $B$ symbols out of $\left[A_{1}^{[k]}, \ldots, A_{n}^{[k]}\right]$ are wrong, the user can 
still successfully decode $\left[X_{1}, \ldots, X_{T}, w_{1}^{[k]}, \ldots, w_{N-2 B-T}^{[k]}\right]$, which include all symbols of $W_{k}$.

It is obvious that database-privacy is guaranteed. Besides $W_{k}$, the user solves $T$ symbols $X_{j}=\left\langle U_{j}, \mathbf{W}\right\rangle+S_{j}$, $j=1, \ldots, T$. Because $S_{1}, \ldots, S_{T}$ are independent uniform symbols drawn from $\mathbb{F}_{q}$, the user can obtain no information about the other $K-1$ messages from the $X_{j}$ 's.

To see that user-privacy is guaranteed, from (12), because $U_{1}, \ldots, U_{T}$ are $T$ independent random vectors and $\mathbf{G}_{\mathbf{U}}$ is the generating matrix of an $(N, T)$-GRS code, any $T$ column vectors of $\mathbf{U G}_{\mathbf{U}}$ are still independent uniform random vectors. Hence, by adding deterministic column vectors of $\mathbf{e G}_{\mathbf{e}}$, any $T$ query vectors are still independent uniform random vectors, and are independent from the desired message index. Therefore, any $T$ colluding servers cannot infer any information about the desired message index.

To conclude, the rate achieved by this scheme is $\frac{N-2 B-T}{N}=$ $1-\frac{2 B+T}{N}$ with secrecy rate $\frac{T}{N-2 B-T}$, which matches the capacity.

\section{B. Converse}

In this section, we prove the converse part of Theorem 1. Lemmas 7-9 below are the versions with colluding servers and replicated databases of Lemmas 2-4 in [10] (and Lemmas 12 in [7]). Hence we merely provide sketches for the proofs. For any set of servers that are not corrupted by the adversary, given their received queries, the answers generated by these servers do not depend on other queries. Because in addition to the received queries, the answers depend on the database and the shared common randomness, which are independent with other queries. Lemma 7 below states that this also holds if conditioned on the requested message.

Lemma 7. For any set of servers $\mathcal{N} \subset[1: N]$ that are not corrupted by the adversary,

$$
H\left(A_{\mathcal{N}}^{[k]} \mid \mathcal{Q}, W_{k}, Q_{\mathcal{N}}^{[k]}\right)=H\left(A_{\mathcal{N}}^{[k]} \mid W_{k}, Q_{\mathcal{N}}^{[k]}\right) .
$$

Proof: We first show that $I\left(A_{\mathcal{N}}^{[k]} ; \mathcal{Q} \mid W_{k}, Q_{\mathcal{N}}^{[k]}\right) \leq 0$, as follows

$$
\begin{aligned}
I\left(A_{\mathcal{N}}^{[k]} ; \mathcal{Q} \mid W_{k}, Q_{\mathcal{N}}^{[k]}\right) & \leq I\left(A_{\mathcal{N}}^{[k]}, W_{[1: K]}, S ; \mathcal{Q} \mid W_{k}, Q_{\mathcal{N}}^{[k]}\right) \\
& \stackrel{(a)}{=} I\left(W_{[1: K]}, S ; \mathcal{Q} \mid W_{k}, Q_{\mathcal{N}}^{[k]}\right) \\
& \leq I\left(W_{[1: K]}, S ; \mathcal{Q}\right)=0
\end{aligned}
$$

where $(a)$ holds because the answers are deterministic functions of the database, common randomness, and the queries. In the last step, $I\left(W_{[1: K]}, S ; \mathcal{Q}\right)=0$ holds because the queries do not depend on the database and common randomness.

On the other hand, it is immediate that $I\left(A_{\mathcal{N}}^{[k]} ; \mathcal{Q} \mid W_{k}, Q_{\mathcal{N}}^{[k]}\right) \geq 0$. Therefore, $H\left(A_{\mathcal{N}}^{[k]} \mid W_{k}, Q_{\mathcal{N}}^{[k]}\right)=$ $H\left(A_{\mathcal{N}}^{[k]} \mid \mathcal{Q}, W_{k}, Q_{\mathcal{N}}^{[k]}\right)$.

Lemma 8. For any set of servers $\mathcal{T} \subset[1: N]$ with size $|\mathcal{T}|=T$ that are not corrupted by the adversary,

$$
\begin{aligned}
H\left(A_{\mathcal{T}}^{[k]} \mid Q_{\mathcal{T}}^{[k]}\right) & =H\left(A_{\mathcal{T}}^{\left[k^{\prime}\right]} \mid Q_{\mathcal{T}}^{\left[k^{\prime}\right]}\right), \\
H\left(A_{\mathcal{T}}^{[k]} \mid W_{k}, Q_{\mathcal{T}}^{[k]}\right) & =H\left(A_{\mathcal{T}}^{\left[k^{\prime}\right]} \mid W_{k}, Q_{\mathcal{T}}^{\left[k^{\prime}\right]}\right) .
\end{aligned}
$$

Proof: The proof is similar as that of Lemma 1 in [7]. We omit the detailed proof here. The key idea is that since any $T$ servers may collude, the statistical distribution of the queries and answers of any $T$ servers shall be the same regardless of the requested message index, even if the servers condition on a part of the database, for example $W_{k}$. Otherwise, the $T$ servers can differentiate between the cases where $W_{k}$ is requested and $W_{k^{\prime}}$ is requested, which contradicts user-privacy (3).

Lemma 9. For any set of servers $\mathcal{T} \subset[1: N]$ with size $|\mathcal{T}|=T$ that are not corrupted by the adversary,

$$
H\left(A_{\mathcal{T}}^{[k]} \mid W_{k}, Q_{\mathcal{T}}^{[k]}\right)=H\left(A_{\mathcal{T}}^{\left[k^{\prime}\right]} \mid Q_{\mathcal{T}}^{\left[k^{\prime}\right]}\right) .
$$

Proof: By database-privacy (4), $I\left(W_{\bar{k}} ; A_{[1: N]}^{\left[k^{\prime}\right]}, \mathcal{Q}\right)=0$. For any $k \neq k^{\prime}$, because $W_{k} \in W_{\bar{k}^{\prime}}$, we have

$$
\begin{aligned}
0 & =I\left(W_{k} ; A_{\mathcal{T}}^{\left[k^{\prime}\right]}, Q_{\mathcal{T}}^{\left[k^{\prime}\right]}\right) \\
& =I\left(W_{k} ; A_{\mathcal{T}}^{\left[k^{\prime}\right]} \mid Q_{\mathcal{T}}^{\left[k^{\prime}\right]}\right)+I\left(W_{k} ; Q_{\mathcal{T}}^{\left[k^{\prime}\right]}\right) \\
& \stackrel{(a)}{=} I\left(W_{k} ; A_{\mathcal{T}}^{\left[k^{\prime}\right]} \mid Q_{\mathcal{T}}^{\left[k^{\prime}\right]}\right) \\
& =H\left(A_{\mathcal{T}}^{\left[k^{\prime}\right]} \mid Q_{\mathcal{T}}^{\left[k^{\prime}\right]}\right)-H\left(A_{\mathcal{T}}^{\left[k^{\prime}\right]} \mid W_{k}, Q_{\mathcal{T}}^{\left[k^{\prime}\right]}\right) \\
& \stackrel{(b)}{=} H\left(A_{\mathcal{T}}^{\left[k^{\prime}\right]} \mid Q_{\mathcal{T}}^{\left[k^{\prime}\right]}\right)-H\left(A_{\mathcal{T}}^{[k]} \mid W_{k}, Q_{\mathcal{T}}^{[k]}\right),
\end{aligned}
$$

where equality $(a)$ holds because $W_{k}$ is independent of the queries, and equality $(b)$ follows by (21).

Lemma 10 below states that the user should be able to decode the desired message from any $N-2 B$ authentic servers. This is similar as Lemma 4 in [1], developed from the cut-set bound in the network coding problem [19], [20], and the distributed storage problem [21]. The difference between our Lemma 10 and Lemma 4 in [1] is that instead of arguing that the answers from any $N-2 B$ authentic servers must be unique for every realization of the database, we argue that it only needs to hold for any realization of the requested message $W_{k}$. For different realizations of the database that differ on messages other than $W_{k}$, the interference may still be the same hence the user can successfully decode. For completeness, we recapitulate the proof of Lemma 4 in [1] with slight modifications for the proof of Lemma 10 below.

Lemma 10. For any set of authentic servers $\mathcal{H} \in[1: N]$ where $|\mathcal{H}|=N-2 B$, for correctly decoding $W_{k}$, the answers $A_{\mathcal{H}}^{[k]}$ are unique for every realization of $W_{k}$. That $i s$, there cannot exist two realizations of the kth message, $W_{k} \neq \tilde{W}_{k}$, such that $A_{\mathcal{H}}^{[k]}\left(W_{k}\right)=A_{\mathcal{H}}^{[k]}\left(\tilde{W}_{k}\right)$. Consequently, $H\left(W_{k} \mid A_{\mathcal{H}}^{[k]}, \mathcal{Q}\right)=0$.

Proof: Divide the $2 B$ servers $[1: N] \backslash \mathcal{H}$ into two size$B$ sets, denoted by $\mathcal{B}_{1}$ and $\mathcal{B}_{2}$. The scheme shall allow the user to correctly decode $W_{k}$ if any $B$ nodes in $[1: N] \backslash \mathcal{H}$ are corrupted by the Byzantine adversary, with any corrupted answers. Consider the following two cases:

- Case 1: The true realization of the $k$ th message is $W_{k}$. The user downloads $A_{\mathcal{H}}^{[k]}\left(W_{k}\right)$ from the authentic servers in $\mathcal{H}$. The servers in $\mathcal{B}_{1}$ are also authentic, generating the answers $A_{\mathcal{B}_{1}}^{[k]}\left(W_{k}\right)$. The servers in $\mathcal{B}_{2}$ are the corrupted servers, the answers from which overwritten by the adversary "happened" to be generated with the agreed scheme 
but by replacing the $k$ th message with $\tilde{W}_{k}$, denoted by $\tilde{A}_{\mathcal{B}_{2}}^{[k]}=A_{\mathcal{B}_{2}}^{[k]}\left(\tilde{W}_{k}\right)$.

- Case 2: Suppose in this case the true realization of the $k$ th message is $\tilde{W}_{k}$, which is different from $W_{k}$ in Case 1. The authentic servers reply to the user based on the true realization of the messages. Therefore, the user downloads $A_{\mathcal{H}}^{[k]}\left(\tilde{W}_{k}\right)$ from $\mathcal{H}$. Suppose that the servers in $\mathcal{B}_{2}$ are also authentic, who send the answers $A_{\mathcal{B}_{2}}^{[k]}\left(\tilde{W}_{k}\right)$ to the user. In Case 2, the $B$ servers in $\mathcal{B}_{1}$ are corrupted. The answers from $\mathcal{B}_{1}$ are generated by replacing the $k$ th message with $W_{k}$, which is the realization of the $k$ th message in Case 1, i.e. $\tilde{A}_{\mathcal{B}_{1}}^{[k]}=A_{\mathcal{B}_{1}}^{[k]}\left(W_{k}\right)$.

If $A_{\mathcal{H}}^{[k]}\left(W_{k}\right)=A_{\mathcal{H}}^{[k]}\left(\tilde{W}_{k}\right)$, under both cases, the user downloads the same set of answers from all servers, i.e., $\quad\left(A_{\mathcal{H}}^{[k]}\left(W_{k}\right)=A_{\mathcal{H}}^{[k]}\left(\tilde{W}_{k}\right), A_{\mathcal{B}_{1}}^{[k]}\left(W_{k}\right), A_{\mathcal{B}_{2}}^{[k]}\left(\tilde{W}_{k}\right)\right)$. Hence, the user cannot successfully decode whether the $k$ th message is $W_{k}$ or $\tilde{W}_{k}$.

In conclusion, for any different realization of $W_{k}$, the answers from $\mathcal{H}$ differs. In other words, the user should be able to successfully decode the desired message from the $N-2 B$ authentic answers, i.e., $H\left(W_{k} \mid A_{\mathcal{H}}^{[k]}, \mathcal{Q}\right)=0$.

1) The proof for $R \leq C_{T-B S P I R}$ : By Lemma 10 , let $\mathcal{H}$ be a set of $N-2 B$ honest servers, $N-2 B \geq T$,

$$
\begin{aligned}
H\left(W_{k}\right) & =H\left(W_{k}\right)-H\left(W_{k} \mid A_{\mathcal{H}}^{[k]}, \mathcal{Q}\right) \\
& =I\left(W_{k} ; A_{\mathcal{H}}^{[k]} \mid \mathcal{Q}\right) \\
& =H\left(A_{\mathcal{H}}^{[k]} \mid \mathcal{Q}\right)-H\left(A_{\mathcal{H}}^{[k]} \mid W_{k}, \mathcal{Q}\right) \\
& \stackrel{(a)}{\leq} H\left(A_{\mathcal{H}}^{[k]} \mid \mathcal{Q}\right)-H\left(A_{\mathcal{T}}^{[k]} \mid W_{k}, \mathcal{Q}\right) \\
& \stackrel{(b)}{=} H\left(A_{\mathcal{H}}^{[k]} \mid \mathcal{Q}\right)-H\left(A_{\mathcal{T}}^{[k]} \mid W_{k}, Q_{\mathcal{T}}^{[k]}\right) \\
& \stackrel{(c)}{=} H\left(A_{\mathcal{H}}^{[k]} \mid \mathcal{Q}\right)-H\left(A_{\mathcal{T}}^{\left[k^{\prime}\right]} \mid Q_{\mathcal{T}}^{\left[k^{\prime}\right]}\right) \\
& \stackrel{(d)}{=} H\left(A_{\mathcal{H}}^{[k]} \mid \mathcal{Q}\right)-H\left(A_{\mathcal{T}}^{[k]} \mid Q_{\mathcal{T}}^{[k]}\right) \\
& \leq H\left(A_{\mathcal{H}}^{[k]} \mid \mathcal{Q}\right)-H\left(A_{\mathcal{T}}^{[k]} \mid \mathcal{Q}\right)
\end{aligned}
$$

In step (a), $\mathcal{T}$ can be any set of $T$ servers in $\mathcal{H}$. Step (b) holds by Lemma 7. Steps (c) and (d) follow by Lemma 9 and Lemma 8 respectively.

Averaging over all $\mathcal{T}$ with size $T$ from $\mathcal{H}$, we have that

$$
H\left(W_{k}\right) \leq H\left(A_{\mathcal{H}}^{[k]} \mid \mathcal{Q}\right)-\frac{1}{\left(\begin{array}{c}
N-2 B \\
T
\end{array}\right)} \sum_{\substack{\mathcal{T} \in \mathcal{H} \\
|\mathcal{T}|=T}} H\left(A_{\mathcal{T}}^{[k]} \mid \mathcal{Q}\right)
$$

By Han's inequality [22],

$$
\frac{1}{\left(\begin{array}{c}
N-2 B \\
T
\end{array}\right)} \sum_{\substack{\mathcal{T} \in \mathcal{H} \\
|\mathcal{T}|=T}} H\left(A_{\mathcal{T}}^{[k]} \mid \mathcal{Q}\right) \geq \frac{T}{N-2 B} H\left(A_{\mathcal{H}}^{[k]} \mid \mathcal{Q}\right) .
$$

Hence, $H\left(W_{k}\right) \leq \frac{N-2 B-T}{N-2 B} H\left(A_{\mathcal{H}}^{[k]} \mid \mathcal{Q}\right) \leq \frac{N-2 B-T}{N-2 B}(N-$ $2 B) H\left(A_{h_{1}}^{[k]} \mid \mathcal{Q}\right) \leq(N-2 B-T) H\left(A_{h_{1}}^{[k]} \mid \mathcal{Q}\right)$, where $h_{1} \in \mathcal{H}$ is an honest server.

Assume that the corrupted servers send the same amount of information bits to the user, otherwise the user can easily identify the corrupted answers. Hence, $R_{\mathrm{T} \text {-BSPIR }}=\frac{H\left(W_{k}\right)}{\sum_{n=1}^{N} H\left(A_{n}^{[k]}\right)}=$ $\frac{H\left(W_{k}\right)}{N \cdot H\left(A_{h_{1}}^{[k]}\right)} \leq 1-\frac{2 B+T}{N}$
2) The proof for $\rho_{T-B S P I R} \geq \frac{T}{N-2 B-T}$ : By database-privacy,

$$
\begin{aligned}
0 & =I\left(W_{\bar{k}} ; A_{\mathcal{H}}^{[k]} \mid \mathcal{Q}\right) \\
= & H\left(W_{\bar{k}} \mid \mathcal{Q}\right)-H\left(W_{\bar{k}} \mid A_{\mathcal{H}}^{[k]}, \mathcal{Q}\right) \\
& \stackrel{(a)}{=} H\left(W_{\bar{k}} \mid \mathcal{Q}, W_{k}\right)-H\left(W_{\bar{k}} \mid A_{\mathcal{H}}^{[k]}, \mathcal{Q}, W_{k}\right) \\
& =I\left(W_{\bar{k}} ; A_{\mathcal{H}}^{[k]} \mid \mathcal{Q}, W_{k}\right) \\
& \stackrel{(b)}{\geq} I\left(W_{\bar{k}} ; A_{\mathcal{T}}^{[k]} \mid \mathcal{Q}, W_{k}\right) \\
& \stackrel{(c)}{=} H\left(A_{\mathcal{T}}^{[k]} \mid \mathcal{Q}, W_{k}\right)-H\left(A_{\mathcal{T}}^{[k]} \mid \mathcal{Q}, W_{[1: K]}\right) \\
& \quad+H\left(A_{\mathcal{T}}^{[k]} \mid \mathcal{Q}, W_{[1: K]}, S\right) \\
= & H\left(A_{\mathcal{T}}^{[k]} \mid \mathcal{Q}, W_{k}\right)-I\left(S ; A_{\mathcal{T}}^{[k]} \mid \mathcal{Q}, W_{[1: K]}\right) \\
\geq & H\left(A_{\mathcal{T}}^{[k]} \mid \mathcal{Q}\right)-H(S),
\end{aligned}
$$

where step (a) follows from Lemma 10 that the user should be able to decode $W_{k}$ from $A_{\mathcal{H}}^{[k]}$. In step (b), $\mathcal{T}$ can be any set of $T$ servers in $\mathcal{H}$. Step (c) holds because the authentic answers are deterministic functions of the queries, the database, and the common randomness.

Averaging over all $\mathcal{T} \subset \mathcal{H}$, and from the proof in Section IV-B1 above,

$$
\begin{aligned}
H(S) & \geq \frac{1}{\left(\begin{array}{c}
N-2 B \\
T
\end{array}\right)} \sum_{\substack{\mathcal{T} \subset \mathcal{H} \\
|\mathcal{T}|=T}} H\left(A_{\mathcal{T}}^{[k]} \mid \mathcal{Q}\right) \\
& \geq \frac{T}{N-2 B} H\left(A_{\mathcal{H}}^{[k]} \mid \mathcal{Q}\right) \\
& \geq \frac{T}{N-2 B-T} H\left(W_{k}\right)
\end{aligned}
$$

Hence, $\rho_{\mathrm{T} \text {-BSPIR }}=\frac{H(S)}{H\left(W_{k}\right)} \geq \frac{T}{N-2 B-T}$.

\section{T-ESPIR}

\section{A. Achievability}

Assume each message comprises $L=N-\max (T, E)$ symbols from a large enough field $\mathbb{F}_{q}$. Let the vector $\mathbf{W}=\left(w_{1}^{[1]}, \ldots, w_{N-\max (T, E)}^{[1]}, \ldots, w_{1}^{[K]}, \ldots, w_{N-\max (T, E)}^{[K]}\right)$ represent the collection of all $K$ messages, which is stored at each server. The user wants to retrieve $W_{k}=$ $\left(w_{1}^{[k]}, \ldots, w_{N-\max (T, E)}^{[k]}\right)$ privately.

The queries are generated in the following way. The user firstly generates $\max (T, E)$ independent uniformly random vectors $U_{1}, \ldots, U_{\max (T, E)}$ of length $K(N-\max (T, E))$ over $\mathbb{F}_{q}$. The user chooses an $(N, \max (T, E))$-GRS code with generating matrix $\mathbf{G}_{(N, \max (T, E))}$. Let $e_{i}^{[k]}$ denote the length$(K(N-\max (T, E)))$ unit vector where only the $((k-1)(N-$ $\max (T, E))+i)$ th entry equals 1 and all the other entries are 0 's. The purpose of $e_{i}^{[k]}$ is to retrieve the $i$ th entry of $W_{k}$. The query vectors are generated by

$$
\begin{aligned}
{\left[Q_{1}^{[k]}, \ldots, Q_{N}^{[k]}\right] } & =\left[U_{1}, \ldots, U_{\max (T, E)}\right] \cdot \mathbf{G}_{(N, \max (T, E))} \\
& +\left[0, \ldots, 0, e_{1}^{[k]}, \ldots, e_{N-\max (T, E)}^{[k]}\right]
\end{aligned}
$$

The nodes share $\max (T, E)$ random symbols $\left(S_{1}, \ldots, S_{\max (T, E)}\right)=\mathbf{S}$, called common randomness, that are uniformly and independently chosen from $\mathbb{F}_{q}$. The common randomness is unavailable to the user and the 
eavesdropper. The servers generate their answers by taking the inner product of the query vector and the stored data vector $\mathbf{W}$, then add on a linear combination of the common randomness in the following way,

$$
A_{n}^{[k]}=\left\langle Q_{n}^{[k]}, \mathbf{W}\right\rangle+\left\langle\mathbf{G}_{(N, \max (T, E))}(n), \mathbf{S}\right\rangle,
$$

where $\mathbf{G}_{(N, \max (T, E))}(n)$ denotes the $n$th column of the matrix $\mathbf{G}_{(N, \max (T, E))}$. Let $X_{j}=\left\langle U_{j}, \mathbf{W}\right\rangle+S_{j}$, where $j=1, \ldots, \max (T, E)$, the answers received by the user can be represented by

$$
\begin{aligned}
{\left[A_{1}^{[k]}, \ldots, A_{N}^{[k]}\right]=} & {\left[X_{1}, \ldots, X_{\max (T, E)}, w_{1}^{[k]}, \ldots, w_{N-\max (T, E)}^{[k]}\right] } \\
& \cdot\left[\begin{array}{c}
\mathbf{G}_{(N, \max (T, E))} \\
\mathbf{0}
\end{array}\right]
\end{aligned}
$$

where we omit the dimension of the zero matrix $\mathbf{0}$ and the identity matrix $\mathbf{I}$ because there is no ambiguity. Because $\mathbf{G}_{(N, \max (T, E))}$ is the generating matrix of an $(N, \max (T, E))$ GRS code, the matrix $\left[\begin{array}{c}\mathbf{G}_{(N, \max (T, E))} \\ \mathbf{0} \mathbf{I}\end{array}\right]$ is invertible. Therefore, from the answers (51), the user can solve the vector $\left[X_{1}, \ldots, X_{\max (T, E)}, w_{1}^{[k]}, \ldots, w_{N-\max (T, E)}^{[k]}\right]$, hence obtain $W_{k}$.

To see that database-privacy is guaranteed, besides the symbols of $W_{k}$, the user solves $\max (T, E)$ random variables $X_{1}, \ldots, X_{\max (T, E)}$, where $X_{j}=\left\langle U_{j}, \mathbf{W}\right\rangle+S_{j}$. Because $S_{1}, \ldots, S_{\max (T, E)}$ are independent uniform symbols drawn from $\mathbb{F}_{q}$, the user can obtain no information about the other $K-1$ messages. User-privacy is also guaranteed, because from equation (49), every $\max (T, E)$ query vectors are independently and uniformly distributed. Hence every $T$ nodes observe independent and uniformly distributed query vectors, no matter which message the user requests. To see that the eavesdropper learns no information about the database, the eavesdropper taps on the queries and answers of $E$ nodes. By the MDS property of GRS codes, any $E$ columns of $\mathbf{G}_{(N, \max (T, E))}$ are linearly independent. From equation (50), any $E$ answers are protected by independent linear combinations of $S_{1}, \ldots, S_{\max (T, E)}$. That is, for any $E$ nodes $n_{1}, \ldots, n_{E},\left\langle\mathbf{G}_{(N, \max (T, E))}\left(n_{i}\right), \mathbf{S}\right\rangle$ 's are statistically independent and uniformly distributed. Hence, from any $E$ query and answer pairs, the eavesdropper obtains no information about the database, i.e. (5) is satisfied.

\section{B. Converse}

In this section, we prove the converse part of Theorem 2. We also use Lemmas 7-9 in Section IV-B for the proofs below.

1) The proof for $R \leq C_{T \text {-ESPIR }}$ : For any message $W_{k}$, $k \in[1: K]$, and any set of nodes $\mathcal{N} \subset[1: N]$ with size $|\mathcal{N}|=\max (T, E)$,

$$
\begin{aligned}
H\left(W_{k}\right) & =H\left(W_{k} \mid \mathcal{Q}\right)-H\left(W_{k} \mid A_{[1: N]}^{[k]}, \mathcal{Q}\right) \\
& =H\left(A_{[1: N]}^{[k]} \mid \mathcal{Q}\right)-H\left(A_{[1: N]}^{[k]} \mid W_{k}, \mathcal{Q}\right) \\
& \leq H\left(A_{[1: N]}^{[k]} \mid \mathcal{Q}\right)-H\left(A_{\mathcal{N}}^{[k]} \mid W_{k}, \mathcal{Q}, Q_{\mathcal{N}}^{[k]}\right) \\
& \stackrel{(a)}{=} H\left(A_{[1: N]}^{[k]} \mid \mathcal{Q}\right)-H\left(A_{\mathcal{N}}^{[k]} \mid W_{k}, Q_{\mathcal{N}}^{[k]}\right) \\
& \stackrel{(b)}{=} H\left(A_{[1: N]}^{[k]} \mid \mathcal{Q}\right)-H\left(A_{\mathcal{N}}^{[k]} \mid Q_{\mathcal{N}}^{[k]}\right)
\end{aligned}
$$

$$
\leq H\left(A_{[1: N]}^{[k]} \mid \mathcal{Q}\right)-H\left(A_{\mathcal{N}}^{[k]} \mid \mathcal{Q}\right)
$$

where $(a)$ holds because given the queries $Q_{\mathcal{N}}^{[k]}$, the answers of $\mathcal{N}$ do not depend on other queries. If $\max (T, E)=T$, by Lemma 9 and Lemma 8 , we have that $(b)$ holds; if $\max (T, E)=E$, from equation (5), $I\left(W_{k} ; A_{\mathcal{N}}^{[k]}, Q_{\mathcal{N}}^{[k]}\right)=0$, hence $(b)$ also holds.

Averaging over all $\mathcal{N}$ with size $\max (T, E)$, we have that

$$
H\left(W_{k}\right) \leq H\left(A_{[1: N]}^{[k]} \mid \mathcal{Q}\right)-\frac{1}{\left(\begin{array}{c}
N \\
\max (T, E)
\end{array}\right.} \sum_{\substack{\mathcal{N} \subset[1: N] \\
|\mathcal{N}|=\max (T, E)}} H\left(A_{\mathcal{N}}^{[k]} \mid \mathcal{Q}\right) .
$$

By Han's inequality [22],

$$
\frac{1}{\left(\begin{array}{c}
N \\
\max (T, E)
\end{array}\right)} \sum_{\substack{\mathcal{N} \in[1: N] \\
|\mathcal{N}|=\max (T, E)}} H\left(A_{\mathcal{N}}^{[k]} \mid \mathcal{Q}\right) \geq \frac{\max (T, E)}{N} H\left(A_{[1: N]}^{[k]} \mid \mathcal{Q}\right) .
$$

Therefore, $R_{\mathrm{T} \text {-ESPIR }}=\frac{H\left(W_{k}\right)}{\sum_{n=1}^{N} H\left(A_{n}^{[k]}\right)} \leq \frac{H\left(W_{k}\right)}{H\left(A_{[1: N]}^{[k]} \mid \mathcal{Q}\right)} \leq 1-$ $\frac{\max (T, E)}{N}$.

2) The proof for $\rho_{T \text {-ESPIR }} \geq \frac{\max (T, E)}{N-\max (T, E)}$ : For any set of nodes $\mathcal{N} \subset[1: N]$ with size $|\mathcal{N}|=\max (T, E)$, from database-privacy (4),

$$
\begin{aligned}
0 & =I\left(W_{\bar{k}} ; A_{[1: N]}^{[k]} \mid \mathcal{Q}\right) \\
= & H\left(W_{\bar{k}} \mid \mathcal{Q}\right)-H\left(W_{\bar{k}} \mid A_{[1: N]}^{[k]}, \mathcal{Q}\right) \\
= & H\left(W_{\bar{k}} \mid \mathcal{Q}, W_{k}\right)-H\left(W_{\bar{k}} \mid A_{[1: N]}^{[k]}, \mathcal{Q}, W_{k}\right) \\
= & I\left(W_{\bar{k}} ; A_{[1: N]}^{[k]} \mid \mathcal{Q}, W_{k}\right) \\
\geq & I\left(W_{\bar{k}} ; A_{\mathcal{N}}^{[k]} \mid \mathcal{Q}, W_{k}\right) \\
& \stackrel{(a)}{=} H\left(A_{\mathcal{N}}^{[k]} \mid \mathcal{Q}, W_{k}\right)-H\left(A_{\mathcal{N}}^{[k]} \mid \mathcal{Q}, W_{[1: K]}\right) \\
& \quad+H^{[k]}\left(A_{\mathcal{N}}^{[k]} \mid \mathcal{Q}, W_{[1: K]}, S\right) \\
= & H\left(A_{\mathcal{N}}^{[k]} \mid \mathcal{Q}, W_{k}\right)-I\left(S ; A_{\mathcal{N}}^{[k]} \mid \mathcal{Q}, W_{[1: K]}\right) \\
\geq & H\left(A_{\mathcal{N}}^{[k]} \mid \mathcal{Q}, W_{k}, Q_{\mathcal{N}}^{[k]}\right)-H(S) \\
& \stackrel{(b)}{=} H\left(A_{\mathcal{N}}^{[k]} \mid Q_{\mathcal{N}}^{[k]}\right)-H(S) \\
\geq & H\left(A_{\mathcal{N}}^{[k]} \mid \mathcal{Q}\right)-H(S) .
\end{aligned}
$$

Equality $(a)$ holds because the answers $A_{\mathcal{N}}^{[k]}$ are deterministic functions of the queries $\mathcal{Q}$, the database $W_{[1: K]}$, and the common randomness $S$. In the proof of the converse part above, we argued that equality $(b)$ holds.

Averaging over all $\mathcal{N}$, and from the proof in Section V-B1 above,

$$
\begin{aligned}
& H(S) \geq \frac{1}{\left(\begin{array}{c}
N \\
\max (T, E)
\end{array}\right.} \sum_{\substack{\mathcal{N} \subset[1: N] \\
|\mathcal{N}|=\max (T, E)}} H\left(A_{\mathcal{N}}^{[k]} \mid \mathcal{Q}\right) \\
& \geq \frac{\max (T, E)}{N} H\left(A_{[1: N]}^{[k]} \mid \mathcal{Q}\right) \\
& \geq \frac{\max (T, E)}{N-\max (T, E)} H\left(W_{k}\right) \text {. }
\end{aligned}
$$

Hence, $\rho_{\mathrm{T} \text {-ESPIR }}=\frac{H(S)}{H\left(W_{k}\right)} \geq \frac{\max (T, E)}{N-\max (T, E)}$. 


\section{T-BESPIR}

The detailed proof of the T-BESPIR capacity (Theorem 3) is omitted. It can be shown by combining the ideas of the proofs for Theorems 1 and 2 . The capacity can be achieved by simply replacing $T$ in the scheme in Section IV-A by $\max (T, E)$. User-privacy, database-privacy and decodability are guaranteed with the same arguments as in Section IV-A. To see that the adversary cannot obtain any information about the database, from (13), every $E$ answers contain linearly independent combinations of $S_{1}, \ldots, S_{\max (T, E)}$, which are uniformly and independently chosen from $\mathbb{F}_{q}$. Therefore, from any $E$ answers, the adversary cannot cancel the $S_{j}$ 's and hence obtains no information about the database.

The converse can be proved by replacing $T$ in the proof of the converse in Section IV-B by $\max (T, E)$, and by replacing the node set $\mathcal{T}$ by any node set $\mathcal{N}$ with $\max (T, E)$ nodes, the same as in Section V-B. Because if $\max (T, E)=T$, by Lemma 8 and Lemma 9, we have that $H\left(A_{\mathcal{N}}^{[k]} \mid W_{k}, Q_{\mathcal{N}}^{[k]}\right)=$ $H\left(A_{\mathcal{N}}^{[k]} \mid Q_{\mathcal{N}}^{[k]}\right)$ holds; if $\max (T, E)=E$, from equation (5), $I\left(W_{k} ; A_{\mathcal{N}}^{[k]}, Q_{\mathcal{N}}^{[k]}\right)=0, H\left(A_{\mathcal{N}}^{[k]} \mid W_{k}, Q_{\mathcal{N}}^{[k]}\right)=H\left(A_{\mathcal{N}}^{[k]} \mid Q_{\mathcal{N}}^{[k]}\right)$ also holds. Therefore, the results are obtained by replacing $T$ in the results of Section IV-B by $\max (T, E)$, that is, $R \leq 1-\frac{2 B+\max (T, E)}{N}$ with $\rho \geq \frac{\max (T, E)}{N-2 B-\max (T, E)}$.

\section{T-EPIR}

\section{A. When $E \geq T$}

The proof of Theorem 4 is direct from the converse proof and achievable scheme for T-ESPIR in Section V, and the complete proofs are provided in our recent work [18], hence the details are omitted and we provide the intuition of the proof as follows. The converse follows by noticing that when $E \geq T$, from the privacy of the database against the eavesdropper (5), $H\left(A_{\mathcal{E}}^{[k]} \mid Q_{\mathcal{E}}^{[k]}\right)=H\left(A_{\mathcal{E}}^{[k]} \mid W_{k}, Q_{\mathcal{E}}^{[k]}\right)$. Lemma 7 still holds for the PIR problem. With similar steps as in Section V-B1, it can be proved that $R \geq 1-\frac{E}{N}$. The achievability follows directly because the scheme in Section V-A which achieves the rate of $1-\frac{E}{N}$ still works for T-EPIR problem (which has one less constraint on the database-privacy). Hence, we can conclude that for $E \geq T$, the capacity of T-EPIR equals $1-\frac{E}{N}$.

\section{B. Inner bound when $E<T$}

In this section, we present an achievable scheme for the case when the eavesdropper can tap in on any $E$ databases where $E<T$. The scheme is developped from the TPIR scheme in [6], by downloading $K$ sectors where each sector uses the scheme in [6] with a different part of the messages and a different part of the common randomness generated by the databases. The three principles in [6] still apply in our scheme.

1) Symmetry across databases

2) Symmetry of message indices within the queries to each database

3) Exploiting the side information of undesired messages to retrieve the desired message information

Specifically, the new ingredient of our scheme lies in iterating the scheme in $K$ sectors to ensure each message is mixed with the common randomness in the same way, hence to fulfill principle 2 . In the following, we firstly present an example in Section VII-B1, and illustrate the decodability, user-privacy and system-privacy. In Section VII-B2, we show the scheme for general parameters of $N, K, T, E$.

We first recapitulate the following lemma from [6]. The lemma states that by multiplying deterministic full rank matrices on uniformly i.i.d. random matrices, the statistics of the random matrices remain unchanged. The proof can be found in [6].

Lemma 11 ( [6]). Let $\mathbf{S}_{1}, \mathbf{S}_{2}, \ldots, \mathbf{S}_{K} \in \mathbb{F}_{q}^{\alpha \times \alpha}$ be $K$ random matrices, drawn independently and uniformly from all $\alpha \times \alpha$ full-rank matrices over $\mathbb{F}_{q}$. Let $\mathbf{G}_{1}, \mathbf{G}_{2}, \ldots, \mathbf{G}_{K} \mathbb{F}_{q}^{\beta \times \beta}$ be $K$ invertible square matrices of dimension $\beta \times \beta$ over $\mathbb{F}_{q}$ where $\beta \leq \alpha$. Let $\mathcal{I}_{1}, \mathcal{I}_{2}, \ldots, \mathcal{I}_{K} \in \mathbb{N}^{1 \times \beta}$ be $K$ index vectors, each containing $\beta$ distinct indices from $[1: \alpha]$, then

$$
\begin{array}{r}
\left(\mathbf{S}_{1}\left[:, \mathcal{I}_{1}\right] \mathbf{G}_{1}, \mathbf{S}_{2}\left[:, \mathcal{I}_{2}\right] \mathbf{G}_{2}, \ldots, \mathbf{S}_{K}\left[:, \mathcal{I}_{K}\right] \mathbf{G}_{K}\right) \sim \\
\quad\left(\mathbf{S}_{1}[:,(1: \beta)], \mathbf{S}_{2}[:,(1: \beta)], \ldots, \mathbf{S}_{K}[:,(1: \beta)]\right)
\end{array}
$$

where $\mathbf{S}_{i}\left[:, \mathcal{I}_{i}\right]$ denotes the $\alpha \times \beta$ matrix comprised of the columns of $\mathbf{S}_{i}$ with indices in $\mathcal{I}_{i}$, and $\sim$ denotes the relation that the random variables on both sides are identically distributed.

1) Example: $N=3$ databases, $K=2$ messages, $T=2$ colluding databases, $E=1$ eavesdropped database: Suppose each message contains $L=13$ symbols from a sufficiently large finite field $\mathbb{F}_{q}, W_{1}=W_{1}^{[1: 13]}$ and $W_{2}=W_{2}^{[1: 13]}$ are represented as length-13 vectors over $\mathbb{F}_{q}$. W.l.o.g., assume the user wants to retrieve $W_{1}$.

The user downloads in two sectors, with 15 symbols in each sector as described in Table III and with detailed formulation below. The databases generate 10 uniformly random symbols, 5 for each sector, denoted as $\left(S_{[1: 5]}^{(1)}, S_{[1: 5]}^{(2)}\right)$. The scheme achieves the rate $R=13 / 30$.

Let $\left\{\lambda_{1}, \ldots, \lambda_{9}\right\}$ be 9 distinct nonzero elements from $\mathbb{F}_{q}$. Let $\mathbf{G}_{[1: 7]}^{7 \times 9}$ and $\mathbf{G}_{[8: 9]}^{2 \times 9}$ be two generating matrices of MDS codes as follows,

$$
\begin{aligned}
\mathbf{G}_{[1: 7]}^{7 \times 9} & =\left[\begin{array}{cccc}
1 & 1 & \ldots & 1 \\
\lambda_{1} & \lambda_{2} & \ldots & \lambda_{9} \\
\vdots & \vdots & \ddots & \vdots \\
\lambda_{1}^{6} & \lambda_{2}^{6} & \ldots & \lambda_{9}^{6}
\end{array}\right], \\
\mathbf{G}_{[8: 9]}^{2 \times 9} & =\left[\begin{array}{cccc}
1 & 1 & \ldots & 1 \\
\lambda_{1} & \lambda_{2} & \ldots & \lambda_{9}
\end{array}\right] \cdot \operatorname{diag}\left(\lambda_{1}^{7}, \lambda_{2}^{7}, \ldots, \lambda_{9}^{7}\right) \\
& =\left[\begin{array}{cccc}
\lambda_{1}^{7} & \lambda_{2}^{7} & \ldots & \lambda_{9}^{7} \\
\lambda_{1}^{8} & \lambda_{2}^{8} & \ldots & \lambda_{9}^{8}
\end{array}\right] .
\end{aligned}
$$

Let $\mathbf{G}=\left[\mathbf{G}_{[1: 7]}^{7 \times 9} \mathbf{G}_{[8: 9]}^{2 \times 9}\right]^{\mathrm{T}}$, then $\mathbf{G}$ is a $9 \times 9$ invertible matrix. Similarly, let $\mathbf{G}_{[1: 6]}^{6 \times 9}$ and $\mathbf{G}_{[7: 9]}^{3 \times 9}$ be composed of the first six rows and the last three rows of $\mathbf{G}$ respectively.

The user privately generates matrices $\mathbf{S}_{1}, \mathbf{S}_{2}, \mathbf{S}_{3}, \mathbf{S}_{4} \in \mathbb{F}_{q}^{9 \times 9}$ uniformly and independently from all $9 \times 9$ invertible matrices over $\mathbb{F}_{q}$.

Let $\mathbf{G}_{1}^{6 \times 9}$ be the generating matrix of a $(9,6)$-MDS code. 


\begin{tabular}{|c|c|c|}
\hline DB1 & DB2 & DB3 \\
\hline$a_{1}^{(r)}, a_{2}^{(r)}$ & $a_{3}^{(r)}, a_{4}^{(r)}$ & $a_{5}^{(r)}, a_{6}^{(r)}$ \\
$b_{1}^{(r)}, b_{2}^{(r)}$ & $b_{3}^{(r)}, b_{4}^{(r)}$ & $b_{5}^{(r)}, b_{6}^{(r)}$ \\
$a_{7}^{(r)}+b_{7}^{(r)}$ & $a_{8}^{(r)}+b_{8}^{(r)}$ & $a_{9}^{(r)}+b_{9}^{(r)}$ \\
\hline
\end{tabular}

TABLE III: The download scheme for each sector $r$, where $r=1$ and $r=2$.

Sector 1:

$$
\begin{gathered}
a_{[1: 9]}^{(1)}=\left(W_{1}^{[1: 7]} \mathbf{G}_{[1: 7]}^{7 \times 9}+\left[S_{1}^{(1)} S_{2}^{(1)}\right] \mathbf{G}_{[8: 9]}^{2 \times 9}\right) \mathbf{S}_{1} \\
b_{[1: 9]}^{(1)}=\left(W_{2}^{[8: 13]} \mathbf{G}_{[1: 6]}^{6 \times 9}+\left[S_{3}^{(1)} S_{4}^{(1)} S_{5}^{(1)}\right] \mathbf{G}_{[7: 9]}^{3 \times 9}\right) \mathbf{S}_{2}[:,(1: 6)] \mathbf{G}_{1}^{6 \times 9}
\end{gathered}
$$

Sector 2:

$$
\begin{gathered}
a_{[1: 9]}^{(2)}=\left(W_{1}^{[8: 13]} \mathbf{G}_{[1: 6]}^{6 \times 9}+\left[S_{3}^{(2)} S_{4}^{(2)} S_{5}^{(2)}\right] \mathbf{G}_{[7: 9]}^{3 \times 9}\right) \mathbf{S}_{3} \\
b_{[1: 9]}^{(2)}=\left(W_{2}^{[1: 7]} \mathbf{G}_{[1: 7]}^{7 \times 9}+\left[S_{1}^{(2)} S_{2}^{(2)}\right] \mathbf{G}_{[8: 9]}^{2 \times 9}\right) \mathbf{S}_{4}[:,(1: 6)] \mathbf{G}_{1}^{6 \times 9}
\end{gathered}
$$

The matrices $\mathbf{G}$ (hence the submatrices $\mathbf{G}_{[1: 7]}^{7 \times 9}$ etc.) and $\mathbf{G}_{1}^{6 \times 9}$ are deterministic and known to the user (can also be known to the databases). The matrices $\mathbf{S}_{1}, \mathbf{S}_{2}, \mathbf{S}_{3}, \mathbf{S}_{4}$ are randomly and privately chosen only at the user's side. The common randomness $\left(S_{[1: 5]}^{(1)}, S_{[1: 5]}^{(2)}\right)$ are generated privately at the databases. After the generation of the $\mathbf{S}$ matrices, the user sends the coefficients of the messages symbols and the common randomness symbols in the linear combinations (75)(78) to the corresponding databases as queries. Upon receiving the queries (the coefficients), the databases calculate the inner product in (75)-(78) and send the results as answers back to the user.

Correctness: In sector 1 , the user can solve $b_{[7: 9]}^{(1)}$ from $b_{[1: 6]}^{(1)}$, because $\mathbf{G}_{1}^{6 \times 9}$ is the generating matrix of a $(9,6)$-MDS code. Therefore, the user can cancel the interference $b_{[7: 9]}^{(1)}$ and obtain $a_{[7: 9]}^{(1)}$. From $a_{[1: 9]}^{(1)}$, the user can solve $W_{1}^{[1: 7]}$, because $a_{[1: 9]}^{(1)}=$ $\left[W_{1}^{[1: 7]} S_{1}^{(1)} S_{2}^{(1)}\right] \mathbf{G S}_{1}$, where $\mathbf{G}$ and $\mathbf{S}_{1}$ are invertible matrices. Similarly in sector 2 , the user can solve $W_{1}^{[8: 13]}$. Hence, the user can solve all 13 symbols of $W_{1}$.

User-privacy: Any $T=2$ databases may collude and observe the queries composed of 6 symbols from $a_{[1: 9]}^{(r)}$ and $b_{[1: 9]}^{(r)}$ for each sector. Let $\mathcal{I}_{a}, \mathcal{I}_{b}$ denote the indices of the symbols observed by the colluding databases,

$$
\begin{aligned}
& \left(a_{\mathcal{I}_{a}}^{(1)}, a_{\mathcal{I}_{a}}^{(2)}, b_{\mathcal{I}_{b}}^{(1)}, b_{\mathcal{I}_{b}}^{(2)}\right) \\
= & \left(\left[W_{1}^{[1: 7]} S_{1}^{(1)} S_{2}^{(1)}\right] \mathbf{G S}_{1}\left[:, \mathcal{I}_{a}\right],\right. \\
& {\left[W_{1}^{[8: 13]} S_{3}^{(2)} S_{4}^{(2)} S_{5}^{(2)}\right] \mathbf{G S}_{3}\left[:, \mathcal{I}_{a}\right], } \\
& {\left[W_{2}^{[8: 13]} S_{3}^{(1)} S_{4}^{(1)} S_{5}^{(1)}\right] \mathbf{G S}_{2}[:,(1: 6)] \mathbf{G}_{1}^{6 \times 9}\left[:, \mathcal{I}_{b}\right], } \\
& {\left.\left[W_{2}^{[1: 7]} S_{1}^{(2)} S_{2}^{(2)}\right] \mathbf{G S}_{4}[:,(1: 6)] \mathbf{G}_{1}^{6 \times 9}\left[:, \mathcal{I}_{b}\right]\right) } \\
\sim & \left(\left[W_{1}^{[1: 7]} S_{1}^{(1)} S_{2}^{(1)}\right] \mathbf{S}_{1}[:,(1: 6)],\right. \\
& {\left[W_{1}^{[8: 13]} S_{3}^{(2)} S_{4}^{(2)} S_{5}^{(2)}\right] \mathbf{S}_{3}[:,(1: 6)], } \\
& {\left[W_{2}^{[8: 13]} S_{3}^{(1)} S_{4}^{(1)} S_{5}^{(1)}\right] \mathbf{S}_{2}[:,(1: 6)], } \\
& {\left.\left[W_{2}^{[1: 7]} S_{1}^{(2)} S_{2}^{(2)}\right] \mathbf{S}_{4}[:,(1: 6)]\right) . }
\end{aligned}
$$

The two sectors of download can be randomized by the user. Therefore, the symbols observed by the two databases are obtained by random mappings from linear combinations of $W_{1}$ and $W_{2}$ and the random symbols $S_{[1: 5]}^{(1)}, S_{[1: 5]}^{(2)}$ generated by the databases in the same way, where the randomness of the mapping is privately generated by the user and unavailable to the databases. Hence, user-privacy is guaranteed.

System-privacy: The eavesdropper can tap in on an arbitrary database. Because the scheme is symmetric across the databases, w.l.o.g., assume DB1 is eavesdropped. In sector 1 , from equation (75) $a_{1}^{(1)}, a_{2}^{(1)}$ are constructed by adding linearly independent combinations of $S_{1}^{(1)}, S_{2}^{(1)}$. Similarly from equation (76), $b_{1}^{(1)}, b_{2}^{(1)}, b_{7}^{(1)}$ are constructed by adding linearly independent combinations of $S_{3}^{(1)}, S_{4}^{(1)}, S_{5}^{(1)}$. Specifically, denote the five answers from DB1 in sector 1 by $A_{D B 1}^{(1)}$, the linear combinations of the $S_{[1: 5]}^{(1)}$ added to the answers are constructed by,

$$
\begin{aligned}
& {\left[S_{1}^{(1)} S_{2}^{(1)} S_{3}^{(1)} S_{4}^{(1)} S_{5}^{(1)}\right] .}
\end{aligned}
$$

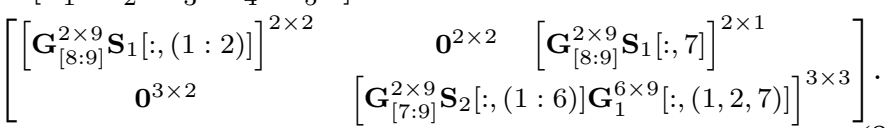

It can be checked that the $5 \times 5$ matrix multiplied to $\left[S_{1}^{(1)} S_{2}^{(1)} S_{3}^{(1)} S_{4}^{(1)} S_{5}^{(1)}\right]$ in $(81)$ is invertible. Therefore, $H\left(A_{D B 1}^{(1)}\right)=H\left(A_{D B 1}^{(1)} \mid W_{1}, W_{2}\right)=5 \log q$. Hence, $I\left(A_{D B 1}^{(1)} ; W_{1}, W_{2}\right)=0$. The construction of symbols for sector 2 are in a similar way, by adding linearly independent combinations of $S_{[1: 5]}^{(2)}$. Because the 10 symbols $S_{[1: 5]}^{(1)}, S_{[1: 5]}^{(2)}$ are independently and uniformly chosen from $\mathbb{F}_{q}$, we have $I\left(A_{D B 1}^{(1)}, A_{D B 1}^{(2)} ; W_{1}, W_{2}\right)=0$ and hence the eavesdropper obtains no information regarding the database $W_{1}, W_{2}$.

2) For arbitrary $N, K, T$ and $E(E<T)$ : Denote $J=\frac{N^{K}-T^{K}}{N-T}$, and suppose each message comprises $L=$ $K N^{K}-E J=K N^{K}-E \frac{N^{K}-T^{K}}{N-T}$ symbols from a large enough finite field. The user downloads $K$ sectors, with $N J$ symbols per sector. The database generates $K E J$ uniformly random symbols, denoted by $S_{[1: E J]}^{(r)}$ where $r=[1: K]$.

Divide $[1: L]$ and $[1: E J]$ into $K$ disjoint sets in the following way,

$$
\begin{gathered}
{[1: L]=} \\
\text { size: } \underbrace{\mathcal{W}_{1}}_{N^{K}-E N^{K-1}} \cup \underbrace{\mathcal{W}_{2}}_{N^{K}-E T N^{K-2}} \cup \cdots \cup \underbrace{\mathcal{W}_{K-1}}_{N^{K}-E T^{K-2}} \cup \underbrace{\mathcal{W}_{K}}_{N^{K}-E T^{K-1}} \\
{[1: E J]=} \\
\underbrace{\mathcal{S}_{1}}_{\text {size: } E N^{K-1}} \cup \underbrace{\mathcal{S}_{2}}_{E T N^{K-2}} \cup \cdots \cup \underbrace{\mathcal{S}_{K-1}}_{E T^{K-2} N} \cup \underbrace{\mathcal{S}_{K}}_{E T^{K-1}}
\end{gathered}
$$

such that $\left|\mathcal{W}_{i}\right|+\left|\mathcal{S}_{i}\right|=N^{K}$. Therefore, $W_{k}^{[1: L]}=$ $\left\{W_{k}^{\mathcal{W}_{1}}, \ldots, W_{k}^{\mathcal{W}_{K}}\right\}$ and $S_{[1: E J]}^{(r)}=\left\{S_{\mathcal{S}_{1}}^{(r)}, \ldots, S_{\mathcal{S}_{K}}^{(r)}\right\}$.

Let $\left\{\lambda_{1}, \ldots, \lambda_{N^{K}}\right\}$ be $N^{K}$ distinct nonzero elements from 
$\mathbb{F}_{q}$. Let $\mathbf{G}$ be a $N^{K} \times N^{K}$ matrix defined as follows,

$$
\mathbf{G}=\left[\begin{array}{cccc}
1 & 1 & \ldots & 1 \\
\lambda_{1} & \lambda_{2} & \ldots & \lambda_{N^{K}} \\
\vdots & \vdots & \ddots & \vdots \\
\lambda_{1}^{N^{K}-1} & \lambda_{2}^{N^{K}-1} & \ldots & \lambda_{N^{K}}^{N^{K}-1}
\end{array}\right]
$$

it can be observed that $\mathbf{G}$ is invertible. In the following, we divide $\mathbf{G}$ into $K$ pairs of matrices $\left\{\mathbf{G}_{\mathcal{W}_{i}}^{\left|\mathcal{W}_{i}\right| \times N^{K}}, \mathbf{G}_{\mathcal{S}_{i}}^{\left|\mathcal{S}_{i}\right| \times N^{K}}\right\}$ for $i=[1: K]$, where $\mathbf{G}_{\mathcal{W}_{i}}^{\left|\mathcal{W}_{i}\right| \times N^{K}}$ is composed of the first $\left|\mathcal{W}_{i}\right|$ rows of $\mathbf{G}$ and $\mathbf{G}_{\mathcal{S}_{i}}^{\left|\mathcal{S}_{i}\right| \times N^{K}}$ is composed of the last $\left|\mathcal{S}_{i}\right|$ rows of $\mathbf{G}$. It is clear that these $2 K$ matrices are generating matrices of MDS codes with corresponding dimensions, and $\mathbf{G}=\left[\mathbf{G}_{\mathcal{W}_{i}}^{\left|\mathcal{W}_{i}\right| \times N^{K}} \mathbf{G}_{\mathcal{S}_{i}}^{\left|\mathcal{S}_{i}\right| \times N^{K}}\right]^{\mathrm{T}}$

For each sector $r$ and each message index $k$, let $V_{k}^{(r)}$ be the length- $N^{K}$ vector defined as follows,

$$
\begin{aligned}
& V_{k}^{(r)}=W_{k}^{\mathcal{W}_{k+r-1 \bmod K}} \mathbf{G}_{\mathcal{W}_{k+r-1 \bmod K}}^{\left|\mathcal{W}_{k+r-1} \bmod \right| \times N^{K}}
\end{aligned}
$$

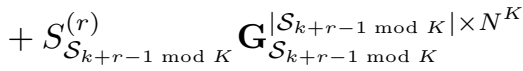

$$
\begin{aligned}
& =\left[W_{k}^{\mathcal{W}_{k+r-1 \bmod K}} S_{\mathcal{S}_{k+r-1 \bmod K}^{(r)}}^{(r)}\right] \mathbf{G},
\end{aligned}
$$

therefore, the $K$ index set pairs $\left(\mathcal{W}_{i}, \mathcal{S}_{i}\right)$ is rotated in all $K$ sector for each message index $k \in[1: K]$. This is to assure user-privacy.

The user privately generates $K^{2}$ matrices $\mathbf{S}_{[1: K]}^{(1)}, \mathbf{S}_{[1: K]}^{(2)}, \ldots, \mathbf{S}_{[1: K]}^{(K)} \in \mathbb{F}_{q}^{N^{K} \times N^{K}}$ uniformly and independently from all $N^{K} \times N^{K}$ invertible matrices over $\mathbb{F}_{q}$.

Suppose the user wants to retrieve $W_{l}$. For any undesired message index $k \in[1: K] \backslash\{l\}$, there are $\Delta=2^{K-2}$ distinct subsets of $[1: K]$ which contain $k$ and do not contain $l$, denoted by $\mathcal{K}_{1}, \mathcal{K}_{2}, \ldots, \mathcal{K}_{\Delta}$. For $i \in[1: \Delta]$, let $\alpha_{i}=N(N-T)^{\left|\mathcal{K}_{i}\right|-1} T^{K-\left|\mathcal{K}_{i}\right|}$, choose $\Delta$ matrices $\mathbf{G}_{1}^{\alpha_{1} \times \frac{N}{T} \alpha_{1}}, \ldots, \mathbf{G}_{\Delta}^{\alpha_{\Delta} \times \frac{N}{T} \alpha_{\Delta}}$ be the generating matrices of the MDS codes with corresponding dimensions.

For each sector $r$, apply the scheme in [6] for $V_{[1: K]}^{(r)}$ as described in (86). For any undesired message index $k \in[1$ : $K] \backslash\{l\}$,

$$
\begin{aligned}
& X_{k}^{(r)} \\
& =\left[\begin{array}{ll:ll:l:ll}
x_{\mathcal{K}_{1}}^{[k],(r)} & x_{\mathcal{K}_{1} \cup\{l\}}^{[k],(r)} & x_{\mathcal{K}_{2}}^{[k],(r)} & x_{\mathcal{K}_{2} \cup\{l\}}^{[k],(r)} & \cdots & x_{\mathcal{K}_{\Delta}}^{[k],(r)} & x_{\mathcal{K}_{\Delta} \cup\{l\}}^{[k],(r)}
\end{array}\right] \\
& =V_{k}^{(r)} \mathbf{S}_{k}^{(r)}\left[:,\left(1: T N^{K-1}\right)\right] \text {. } \\
& {\left[\begin{array}{cccc}
\mathbf{G}_{1}^{\alpha_{1} \times \frac{N}{T} \alpha_{1}} & \mathbf{0} & \cdots & \mathbf{0} \\
\mathbf{0} & \mathbf{G}_{2}^{\alpha_{2} \times \frac{N}{T} \alpha_{2}} & \cdots & \mathbf{0} \\
\vdots & \vdots & \vdots & \vdots \\
\mathbf{0} & \mathbf{0} & \mathbf{0} & \mathbf{G}_{\Delta}^{\alpha_{\Delta} \times \frac{N}{T} \alpha_{\Delta}}
\end{array}\right],}
\end{aligned}
$$

where the length of $x_{\mathcal{K}_{i},}^{[k],(r)}$ is $\alpha_{i}=N(N-T)^{\left|\mathcal{K}_{i}\right|-1} T^{K-\left|\mathcal{K}_{i}\right|}$ and the length of $x_{\mathcal{K}_{i} \cup\{l\}}^{[k],(r)}$ is $\frac{N-T}{T} \alpha_{i}$.

For the desired message index $l$, there are $\delta=2^{K-1}$ distinct subsets of $[1: K]$ which contain $l$, denoted by $\mathcal{L}_{1}, \mathcal{L}_{2}, \ldots, \mathcal{L}_{\delta}$.
Let

$$
X_{l}^{(r)}=\left[\begin{array}{llll}
x_{\mathcal{L}_{1}}^{[l],(r)} & x_{\mathcal{L}_{2}}^{[l],(r)} & \cdots & x_{\mathcal{L}_{\delta}}^{[l],(r)}
\end{array}\right]=V_{l}^{(r)} \mathbf{S}_{l}^{(r)},
$$

where the length of $x_{\mathcal{L}_{i}}^{[l],(r)}$ is $N(N-T)^{\left|\mathcal{L}_{i}\right|-1} T^{K-\left|\mathcal{L}_{i}\right|}$.

For each non-empty set $\mathcal{K} \in[1: K]$, the queries associated with $\mathcal{K}$ is generated by

$$
\mathcal{Q}_{\mathcal{K}}^{(r)}=\sum_{k \in \mathcal{K}} x_{\mathcal{K}}^{(r)}
$$

For all $K$ sectors $r \in[1: K]$, distribute the queries for each $\mathcal{K}$ evenly among the $N$ databases, and the construction of the queries is completed.

Decodability, User-privacy, System-privacy, and the Achievable rate

From [6], for each sector, the user can cancel the interference of the undesired messages hence obtain $V_{l}^{(r)}$ for all $K$ sectors. Furthermore, from (86), the user can solve for a different set of symbols $W_{l}^{\mathcal{W}_{i}}$ each sector, hence the user can obtain all the symbols of the desired message $W_{l}^{[1: L]}=$ $\left\{W_{l}^{\mathcal{W}_{1}}, \ldots, W_{l}^{\mathcal{W}_{K}}\right\}$.

To see why user-privacy is guaranteed, similarly as in [6], any $T$ colluding servers observe queries comprised of $T N^{K-1}$ symbols of $X_{k}^{(r)}$ for each sector. Denote the index set of $X_{k}^{(r)}$ observed by the colluding servers by $\mathcal{I}_{k}$, we have that for all $k \in[1: K]$,

$$
X_{\mathcal{I}_{k}}^{(r)} \sim V_{k}^{(r)} \mathbf{S}_{k}^{(r)}\left[:,\left(1: T N^{K-1}\right)\right] .
$$

From (86), $V_{k}^{(r)}$ are constructed from disjoint sets of symbols of $W_{k}$ in an iterative manner through the $K$ sectors, and because $\mathbf{S}_{k}^{(r)}\left[:,\left(1: T N^{K-1}\right)\right]$ are independently and identically distributed, user-privacy is guaranteed since the colluding databases observe symbols constructed from all $W_{k}$ 's through the same random mapping.

System-privacy is guaranteed due to (83) and (86), for each sector the $E J$ queries and answers observed by the eavesdropper is constructed by adding independent linear combinations of $E J$ independent uniform symbols $S_{[1: E J]}^{(r)}$ : Therefore, the eavesdropper can obtain no information regarding the database $W_{[1: K]}$.

The rate achieved by the scheme is

$$
R=\frac{L}{K N J}=\frac{K N^{K}-E \frac{N^{K}-T^{K}}{N-T}}{K N \frac{N^{K}-T^{K}}{N-T}}=\frac{1-\frac{T}{N}}{1-\left(\frac{T}{N}\right)^{K}}-\frac{E}{K N} .
$$

The secrecy rate achieved is

$$
\rho=\frac{K E J}{L}=\frac{K E \frac{N^{K}-T^{K}}{N-T}}{K N^{K}-E \frac{N^{K}-T^{K}}{N-T}}=\frac{\frac{E}{N}\left(1-\left(\frac{T}{N}\right)^{K}\right)}{1-\frac{T}{N}-\frac{E}{K N}\left(1-\left(\frac{T}{N}\right)^{K}\right)} .
$$

\section{Outer bound when $E<T$}

In this section, we derive the outer bound presented in Theorem 5 for the PIR problem with $T$-colluding databases and $E$-eavesdropped databases when $E<T$. When $K=1$, userprivacy is guaranteed directly. To prevent the eavesdropper from learning the database, $E$ out of $N$ answers cannot provide any useful information on the database. Therefore, it is direct that an outer bound on the retrieval rate is $1-\frac{E}{N}$. In the 
following, we start from the case when $K=2$, then generalize to the case of arbitrary $K$ messages.

1) $K=2$ Messages: For any set of nodes $\mathcal{T} \subset[1: N]$ with $|\mathcal{T}|=T$,

$$
\begin{aligned}
L=H\left(W_{1}\right) & =H\left(W_{1} \mid \mathcal{Q}\right)-H\left(W_{1} \mid A_{[1: N]}^{[1]}, \mathcal{Q}\right) \\
& =H\left(A_{[1: N]}^{[1]} \mid \mathcal{Q}\right)-H\left(A_{[1: N]}^{[1]} \mid W_{1}, \mathcal{Q}\right) \\
& \leq H\left(A_{[1: N]}^{[1]} \mid \mathcal{Q}\right)-H\left(A_{\mathcal{T}}^{[1]} \mid W_{1}, \mathcal{Q}\right) \\
& =H\left(A_{[1: N]}^{[1]} \mid \mathcal{Q}\right)-H\left(A_{\mathcal{T}}^{[2]} \mid W_{1}, \mathcal{Q}\right) \\
& \leq H\left(A_{[1: N]}^{[1]} \mid \mathcal{Q}\right)-\frac{T}{N} H\left(A_{[1: N]}^{[2]} \mid W_{1}, \mathcal{Q}\right),
\end{aligned}
$$

where (91) follows from decodability (2) and the fact that messages are independent of queries. Step (94) holds because by user-privacy (3), any set of servers $\mathcal{T}$ should not be able to distinguish between different desired message indices even by fixing part of the database. The last step (95) follows by averaging over all set $\mathcal{T} \subset[1: N]$ with $|\mathcal{T}|=T$, and by applying Han's inequality [22]. Hence, we have that

$$
H\left(A_{[1: N]}^{[2]} \mid W_{1}, \mathcal{Q}\right) \leq \frac{N}{T}\left(H\left(A_{[1: N]}^{[1]} \mid \mathcal{Q}\right)-L\right) .
$$

For any set of nodes $\mathcal{E} \subset[1: N]$ with $|\mathcal{E}|=E$, and any set of nodes $\mathcal{T} \subset[1: N]$ with $|\mathcal{T}|=T$,

$$
\begin{aligned}
2 L= & H\left(W_{1}, W_{2}\right) \\
= & H\left(W_{1}, W_{2} \mid \mathcal{Q}\right)-H\left(W_{1}, W_{2} \mid A_{[1: N]}^{[1]}, A_{[1: N]}^{[2]}, \mathcal{Q}\right) \\
= & H\left(A_{[1: N]}^{[1]}, A_{[1: N]}^{[2]} \mid \mathcal{Q}\right)-H\left(A_{[1: N]}^{[1]}, A_{[1: N]}^{[2]} \mid W_{1}, W_{2}, \mathcal{Q}\right) \\
\leq & H\left(A_{[1: N]}^{[1]}, A_{[1: N]}^{[2]} \mid \mathcal{Q}\right)-H\left(A_{\mathcal{E}} \mid W_{1}, W_{2}, \mathcal{Q}\right) \\
= & H\left(A_{[1: N]}^{[1]}, A_{[1: N]}^{[2]} \mid \mathcal{Q}\right)-H\left(A_{\mathcal{E}} \mid \mathcal{Q}\right) \\
= & H\left(A_{[1: N]}^{[1]} \mid \mathcal{Q}\right)+H\left(A_{[1: N]}^{[2]} \mid A_{[1: N]}^{[1]}, W_{1}, \mathcal{Q}\right)-H\left(A_{\mathcal{E}} \mid \mathcal{Q}\right) \\
\leq & H\left(A_{[1: N]}^{[1]} \mid \mathcal{Q}\right)+H\left(A_{[1: N]}^{[2]} \mid A_{\mathcal{T}}, W_{1}, \mathcal{Q}\right)-H\left(A_{\mathcal{E}} \mid \mathcal{Q}\right) \\
= & H\left(A_{[1: N]}^{[1]} \mid \mathcal{Q}\right)+H\left(A_{[1: N]}^{[2]} \mid W_{1}, \mathcal{Q}\right)-H\left(A_{\mathcal{T}} \mid W_{1}, \mathcal{Q}\right) \\
& \quad-H\left(A_{\mathcal{E}} \mid \mathcal{Q}\right) \\
\leq & H\left(A_{[1: N]}^{[1]} \mid \mathcal{Q}\right)+\left(1-\frac{T}{N}\right) H\left(A_{[1: N]}^{[2]} \mid W_{1}, \mathcal{Q}\right) \\
& \quad-H\left(A_{\mathcal{E}} \mid \mathcal{Q}\right) \\
\leq & H\left(A_{[1: N]}^{[1]} \mid \mathcal{Q}\right)+\left(1-\frac{T}{N}\right) \frac{N}{T}\left(H\left(A_{[1: N]}^{[1]} \mid \mathcal{Q}\right)-L\right) \\
& \quad-H\left(A_{\mathcal{E}} \mid \mathcal{Q}\right) \\
\leq & H\left(A_{[1: N]}^{[1]} \mid \mathcal{Q}\right)+\left(1-\frac{T}{N}\right) \frac{N}{T}\left(H\left(A_{[1: N]}^{[1]} \mid \mathcal{Q}\right)-L\right) \\
& \quad-\frac{E}{N} H\left(A_{[1: N]}^{[1]} \mid \mathcal{Q}\right) \\
= & \left(\frac{N}{T}-\frac{E}{N}\right) H\left(A_{[1: N]}^{[1]} \mid \mathcal{Q}\right)-\left(\frac{N}{T}-1\right) L,
\end{aligned}
$$

where in (100) we can omit the message index because $\mathcal{E}$ is a set with size $E \leq T$. (101) follows from systemprivacy (5). (102) is due to the fact that the user can decode
$W_{1}$ from $A_{[1: N]}^{[1]}$ and $\mathcal{Q}$. (105) is obtained by averaging over all $\mathcal{T}$ with size $T$ and applying Han's inequality. (106) follows from (96). (107) is obtained by averaging over all $\mathcal{E}$ with size $E$ and applying Han's inequality.

Therefore, we have that $\left(\frac{N}{T}-\frac{E}{N}\right) H\left(A_{[1: N]}^{[1]} \mid \mathcal{Q}\right) \geq$ $\left(\frac{N}{T}+1\right) L$ and

$$
R=\frac{L}{\sum_{n=1}^{N} H\left(A_{n}^{[1]}\right)} \leq \frac{L}{H\left(A_{[1: N]}^{[1]} \mid \mathcal{Q}\right)} \leq \frac{1-\frac{E}{N} \cdot \frac{T}{N}}{1+\frac{T}{N}} .
$$

2) $K \geq 3$ Messages: For any set of nodes $\mathcal{T} \subset[1: N]$ with $|\mathcal{T}|=T$, and its compliment set $\overline{\mathcal{T}}=[1: N] \backslash \mathcal{T}$, and for any $k \in[2: K]$,

$$
\begin{aligned}
& H\left(A_{\overline{\mathcal{T}}}^{[k]} \mid A_{\mathcal{T}}, W_{[1: k-1]}, \mathcal{Q}\right) \\
= & H\left(A_{[1: N]}^{[k]} \mid W_{[1: k-1]}, \mathcal{Q}\right)-H\left(A_{\mathcal{T}} \mid W_{[1: k-1]}, \mathcal{Q}\right) \\
\leq & \left(1-\frac{T}{N}\right) H\left(A_{[1: N]}^{[k]} \mid W_{[1: k-1]}, \mathcal{Q}\right),
\end{aligned}
$$

where the last step follows by averaging over all $\mathcal{T}$ with size $T$ and applying Han's inequality [22].

From $A_{[1: N]}^{[1]}, \ldots, A_{[1: N]}^{[k-1]}$, the user can decode $W_{[1: k-1]}$, hence

$$
\begin{aligned}
& (k-1) L \\
= & I\left(W_{[1: k-1]} ; A_{[1: N]}^{[1]}, \ldots, A_{[1: N]}^{[k-1]} \mid \mathcal{Q}\right) \\
= & H\left(A_{[1: N]}^{[1]}, \ldots, A_{[1: N]}^{[k-1]} \mid \mathcal{Q}\right)- \\
& H\left(A_{[1: N]}^{[1]}, \ldots, A_{[1: N]}^{[k-1]} \mid W_{[1: k-1]}, \mathcal{Q}\right) \\
\leq & H\left(A_{[1: N]}^{[1]}, \ldots, A_{[1: N]}^{[k-1]} \mid \mathcal{Q}\right)-H\left(A_{\mathcal{T}} \mid W_{[1: k-1]}, \mathcal{Q}\right) \\
\leq & H\left(A_{[1: N]}^{[1]}, \ldots, A_{[1: N]}^{[k-1]} \mid \mathcal{Q}\right)-\frac{T}{N} H\left(A_{[1: N]}^{[k]} \mid W_{[1: k-1]}, \mathcal{Q}\right),
\end{aligned}
$$

where in (116), we can omit the message index of $A_{\mathcal{T}}$ because from user-privacy (3), the answers of any $T$ databases are independent of the message index. Similar as above, the last step follows by averaging over all $\mathcal{T}$ with size $T$ and applying Han's inequality [22]. Because $A_{\mathcal{T}}$ is independent of the message index, we can set the desired message index to be $k$ in the last step (117).

Therefore, from (112) and (117), for any $k \in[2: K]$,

$$
\begin{aligned}
& H\left(A_{\overline{\mathcal{T}}}^{[k]} \mid A_{\mathcal{T}}, W_{[1: k-1]}, \mathcal{Q}\right) \\
\leq & \left(1-\frac{T}{N}\right) H\left(A_{[1: N]}^{[k]} \mid W_{[1: k-1]}, \mathcal{Q}\right) \\
\leq & \left(1-\frac{T}{N}\right) \frac{N}{T}\left(H\left(A_{[1: N]}^{[1]}, \ldots, A_{[1: N]}^{[k-1]} \mid \mathcal{Q}\right)-(k-1) L\right) \\
= & \left(\frac{N}{T}-1\right)\left(H\left(A_{\mathcal{T}}, A_{\mathcal{T}}^{[1]}, \ldots, A_{\overline{\mathcal{T}}}^{[k-1]} \mid \mathcal{Q}\right)-(k-1) L\right) \\
= & \left(\frac{N}{T}-1\right)\left(H\left(A_{[1: N]}^{[1]} \mid \mathcal{Q}\right)+\right. \\
& \left.H\left(A_{\overline{\mathcal{T}}}^{[2]}, \ldots, A_{\overline{\mathcal{T}}}^{[k-1]} \mid A_{\mathcal{T}}, A_{\overline{\mathcal{T}}}^{[1]}, W_{1}, \mathcal{Q}\right)-(k-1) L\right)
\end{aligned}
$$




$$
\begin{aligned}
\leq & \left(\frac{N}{T}-1\right)\left(H\left(A_{[1: N]}^{[1]} \mid \mathcal{Q}\right)+\right. \\
& \left.H\left(A_{\overline{\mathcal{T}}}^{[2]}, \ldots, A_{\overline{\mathcal{T}}}^{[k-1]} \mid A_{\mathcal{T}}, W_{1}, \mathcal{Q}\right)-(k-1) L\right) \\
\leq & \left(\frac{N}{T}-1\right)\left(H\left(A_{[1: N]}^{[1]} \mid \mathcal{Q}\right)+H\left(A_{\mathcal{\mathcal { T }}}^{[2]} \mid A_{\mathcal{T}}, W_{1}, \mathcal{Q}\right)+\cdots\right. \\
& \left.+H\left(A_{\overline{\mathcal{T}}}^{[k-1]} \mid A_{\mathcal{T}}, W_{[1: k-2]}, \mathcal{Q}\right)-(k-1) L\right)
\end{aligned}
$$

where (122) holds because from $A_{\mathcal{T}}, A_{\overline{\mathcal{T}}}^{[1]}$ and $\mathcal{Q}$ one can decode $W_{1}$. The last step is obtained by repeating the chain rule and by the fact that from $A_{\mathcal{T}}, A_{\overline{\mathcal{T}}}^{[i]}$ and $\mathcal{Q}$ one can decode $W_{i}$ for $i=[2: k-2]$.

For any set of nodes $\mathcal{E} \subset[1: N]$ with $|\mathcal{E}|=E$,

$$
\begin{aligned}
& K L=H\left(W_{[1: K]}\right) \\
& =I\left(W_{[1: K]} ; A_{\mathcal{T}}, A_{\overline{\mathcal{T}}}^{[1]}, \ldots, A_{\overline{\mathcal{T}}}^{[K]} \mid \mathcal{Q}\right) \\
& =H\left(A_{\mathcal{T}}, A_{\overline{\mathcal{T}}}^{[1]}, \ldots, A_{\overline{\mathcal{T}}}^{[K]} \mid \mathcal{Q}\right) \\
& -H\left(A_{\mathcal{T}}, A_{\overline{\mathcal{T}}}^{[1]}, \ldots, A_{\overline{\mathcal{T}}}^{[K]} \mid W_{[1: K]}, \mathcal{Q}\right) \\
& \leq H\left(A_{\mathcal{T}}, A_{\overline{\mathcal{T}}}^{[1]}, \ldots, A_{\overline{\mathcal{T}}}^{[K]} \mid \mathcal{Q}\right)-H\left(A_{\mathcal{E}} \mid W_{[1: K]}, \mathcal{Q}\right) \\
& =H\left(A_{\mathcal{T}}, A_{\overline{\mathcal{T}}}^{[1]}, \ldots, A_{\overline{\mathcal{T}}}^{[K]} \mid \mathcal{Q}\right)-H\left(A_{\mathcal{E}} \mid \mathcal{Q}\right) \\
& =H\left(A_{[1: N]}^{[1]} \mid \mathcal{Q}\right)+H\left(A_{\overline{\mathcal{T}}}^{[2]}, \ldots, A_{\overline{\mathcal{T}}}^{[K]} \mid A_{[1: N]}^{[1]}, \mathcal{Q}\right)-H\left(A_{\mathcal{E}} \mid \mathcal{Q}\right) \\
& =H\left(A_{[1: N]}^{[1]} \mid \mathcal{Q}\right)+H\left(A_{\overline{\mathcal{T}}}^{[2]}, \ldots, A_{\overline{\mathcal{T}}}^{[K]} \mid A_{[1: N]}^{[1]}, W_{1}, \mathcal{Q}\right) \\
& -H\left(A_{\mathcal{E}} \mid \mathcal{Q}\right) \\
& \leq H\left(A_{[1: N]}^{[1]} \mid \mathcal{Q}\right)+H\left(A_{\overline{\mathcal{T}}}^{[2]}, \ldots, A_{\overline{\mathcal{T}}}^{[K]} \mid A_{\mathcal{T}}, W_{1}, \mathcal{Q}\right)-H\left(A_{\mathcal{E}} \mid \mathcal{Q}\right) \\
& \leq H\left(A_{[1: N]}^{[1]} \mid \mathcal{Q}\right)+H\left(A_{\overline{\mathcal{T}}}^{[2]} \mid A_{\mathcal{T}}, W_{1}, \mathcal{Q}\right)+ \\
& H\left(A_{\overline{\mathcal{T}}}^{[3]}, \mid A_{\mathcal{T}}, W_{[1: 2]}, \mathcal{Q}\right)+\cdots+H\left(A_{\overline{\mathcal{T}}}^{[K]}, \mid A_{\mathcal{T}}, W_{[1: K-1]}, \mathcal{Q}\right) \\
& \text { - } H\left(A_{\mathcal{E}} \mid \mathcal{Q}\right)
\end{aligned}
$$$$
\leq H\left(A_{[1: N]}^{[1]} \mid \mathcal{Q}\right)+H\left(A_{\overline{\mathcal{T}}}^{[2]} \mid A_{\mathcal{T}}, W_{1}, \mathcal{Q}\right)+
$$$$
H\left(A_{\overline{\mathcal{T}}}^{[3]}, \mid A_{\mathcal{T}}, W_{[1: 2]}, \mathcal{Q}\right)+\cdots+\left(\frac{N}{T}-1\right)\left(H\left(A_{[1: N]}^{[1]} \mid \mathcal{Q}\right)\right.
$$$$
+H\left(A_{\overline{\mathcal{T}}}^{[2]} \mid A_{\mathcal{T}}, W_{1}, \mathcal{Q}\right)+\cdots+H\left(A_{\overline{\mathcal{T}}}^{[K-1]} \mid A_{\mathcal{T}}, W_{[1: K-2]}, \mathcal{Q}\right)
$$$$
-(K-1) L)-H\left(A_{\mathcal{E}} \mid \mathcal{Q}\right)
$$$$
=\frac{N}{T}\left(H\left(A_{[1: N]}^{[1]} \mid \mathcal{Q}\right)+H\left(A_{\overline{\mathcal{T}}}^{[2]} \mid A_{\mathcal{T}}, W_{1}, \mathcal{Q}\right)+\cdots+\right.
$$$$
\left.H\left(A_{\overline{\mathcal{T}}}^{[K-1]} \mid A_{\mathcal{T}}, W_{[1: K-2]}, \mathcal{Q}\right)\right)-\left(\frac{N}{T}-1\right)(K-1) L
$$$$
-H\left(A_{\mathcal{E}} \mid \mathcal{Q}\right)
$$$$
\leq\left(\frac{N}{T}\right)^{K-1} H\left(A_{[1: N]}^{[1]} \mid \mathcal{Q}\right)-H\left(A_{\mathcal{E}} \mid \mathcal{Q}\right)-\left(1-\frac{T}{N}\right) \text {. }
$$$$
\left[\frac{N}{T}(K-1) L+\left(\frac{N}{T}\right)^{2}(K-2) L+\cdots+\left(\frac{N}{T}\right)^{K-1} L\right]
$$$$
=\left(\frac{N}{T}\right)^{K-1} H\left(A_{[1: N]}^{[1]} \mid \mathcal{Q}\right)-H\left(A_{\mathcal{E}} \mid \mathcal{Q}\right)
$$$$
-\frac{\left(\frac{N}{T}\right)^{K}-\frac{N}{T}}{\frac{N}{T}-1} L+(K-1) L
$$

$$
\begin{aligned}
\leq & \left(\left(\frac{N}{T}\right)^{K-1}-\frac{E}{N}\right) H\left(A_{[1: N]}^{[1]} \mid \mathcal{Q}\right)-\frac{\left(\frac{N}{T}\right)^{K}-\frac{N}{T}}{\frac{N}{T}-1} L \\
& +(K-1) L,
\end{aligned}
$$

where (129) is due to system-privacy (5). Steps from (130)(133) follow by repeating the chain rule and by the fact that from $A_{\mathcal{T}}, A_{\overline{\mathcal{T}}}^{[i]}$ and $\mathcal{Q}$ one can decode $W_{i}$ for $i=[1: K-$ 1]. Step (134) follows by using inequality (124) for $k=K$. By iteratively using inequality (124) for $k=\{K-1, K-$ $2, \ldots, 2\}$, we obtain (136). The last step follows by averaging over all $\mathcal{E}$ with size $E$ and applying Han's inequality.

Therefore, $\left(\left(\frac{N}{T}\right)^{K-1}-\frac{E}{N}\right) H\left(A_{[1: N]}^{[1]} \mid \mathcal{Q}\right) \geq \frac{\left(\frac{N}{T}\right)^{K}-1}{\frac{N}{T}-1} L$, and hence

$$
\begin{aligned}
R=\frac{L}{\sum_{n=1}^{N} H\left(A_{n}^{[1]}\right)} & \leq \frac{L}{H\left(A_{[1: N]}^{[1]} \mid \mathcal{Q}\right)} \\
& \leq\left(\frac{N}{T}-1\right) \frac{\left(\frac{N}{T}\right)^{K-1}-\frac{E}{N}}{\left(\frac{N}{T}\right)^{K}-1} \\
& =\left(1-\frac{T}{N}\right) \frac{1-\frac{E}{N} \cdot\left(\frac{T}{N}\right)^{K-1}}{1-\left(\frac{T}{N}\right)^{K}} \\
& =\bar{R}_{\text {T-EPIR. }}
\end{aligned}
$$

To obtain a lower bound on the amount of common randomness needed to guarantee system-privacy, for any set of nodes $\mathcal{E} \subset[1: N]$ with size $|\mathcal{E}|=E$,

$$
\begin{aligned}
0 & =I\left(A_{\mathcal{E}} ; W_{[1: K]} \mid \mathcal{Q}\right) \\
& =H\left(A_{\mathcal{E}} \mid \mathcal{Q}\right)-H\left(A_{\mathcal{E}} \mid W_{[1: K]}, \mathcal{Q}\right) \\
& =H\left(A_{\mathcal{E}} \mid \mathcal{Q}\right)-H\left(A_{\mathcal{E}} \mid W_{[1: K]}, \mathcal{Q}\right)+H\left(A_{\mathcal{E}} \mid W_{[1: K]}, S, \mathcal{Q}\right) \\
& =H\left(A_{\mathcal{E}} \mid \mathcal{Q}\right)-I\left(S ; A_{\mathcal{E}} \mid W_{[1: K]}, \mathcal{Q}\right) \\
& =H\left(A_{\mathcal{E}} \mid \mathcal{Q}\right)-H\left(S \mid W_{[1: K]}, \mathcal{Q}\right)+H\left(S \mid A_{\mathcal{E}}, W_{[1: K]}, \mathcal{Q}\right) \\
& \geq H\left(A_{\mathcal{E}} \mid \mathcal{Q}\right)-H(S),
\end{aligned}
$$

where (145) holds because $A_{\mathcal{E}}$ is a deterministic function of $W_{[1: K]}, S$ and $\mathcal{Q}$. By averaging over all $\mathcal{E}$ with size $E$ and applying Han's inequality [22],

$$
\begin{aligned}
H(S) & \geq \frac{1}{\left(\begin{array}{c}
N \\
E
\end{array}\right)} \sum_{\substack{\mathcal{E} \subset[1: N] \\
|\mathcal{E}|=E}} H\left(A_{\mathcal{E}} \mid \mathcal{Q}\right) \geq \frac{E}{N} H\left(A_{[1: N]}^{[1]} \mid \mathcal{Q}\right) \\
& \geq \frac{\frac{E}{N}\left(1-\left(\frac{T}{N}\right)^{K}\right)}{\left(1-\frac{T}{N}\right)\left(1-\frac{E}{N} \cdot\left(\frac{T}{N}\right)^{K-1}\right)} L .
\end{aligned}
$$

Therefore, $\rho_{\text {T-EPIR }}=\frac{H(S)}{L} \geq \frac{\frac{E}{N}\left(1-\left(\frac{T}{N}\right)^{K}\right)}{\left(1-\frac{T}{N}\right)\left(1-\frac{E}{N} \cdot\left(\frac{T}{N}\right)^{K-1}\right)}$.

Remark: After the submission of this manuscript, we characterize the T-EPIR capacity in our recent work [18]. We keep the derivation of the bounds in this work because the techniques might be of interest for future studies.

\section{CONCLUSION}

In this paper, we study several variants of the PIR and SPIR problems with colluding databases, where the system 
is vulnerable to adversaries and/or eavesdroppers. The main contribution of this work is the characterization of the SPIR capacity with $T$-colluding databases in three scenarios: with an Byzantine adversary; with an eavesdropper; and with an adversary who can both eavesdrop and corrupt communications. We also study the $T$-colluding PIR problem with an eavesdropper. When $E \geq T$, the capacity is established, which is shown to be equal to the T-ESPIR capacity. Therefore, in this case, there is no extra penalty in requiring further database-privacy at the user's side. When $E<T$, we derive outer and inner bounds on the optimal achievable rate. In our recent work [18], we improve on the bounds and settle the T-EPIR capacity.

Regarding future work, one interesting direction is to investigate the information-theoretic limits of the problem where the eavesdropper can also tap in on the storage at the servers (schemes are provided in a recent work [23]). The other promising direction is to explore the $T$-colluding PIR problem with a combination adversary who can both eavesdrop and corrupt communications.

\section{REFERENCES}

[1] K. Banawan and S. Ulukus, "The capacity of private information retrieval from Byzantine and colluding databases," arXiv preprint arXiv:1706.01442, 2017.

[2] B. Chor, O. Goldreich, E. Kushilevitz, and M. Sudan, "Private information retrieval," in IEEE Annual Symposium on Foundations of Computer Science, 1995, pp. 41-50.

[3] B. Chor, E. Kushilevitz, O. Goldreich, and M. Sudan, "Private information retrieval," Journal of the ACM (JACM), 1998.

[4] Y. Gertner, Y. Ishai, E. Kushilevitz, and T. Malkin, "Protecting data privacy in private information retrieval schemes," in Proceedings of the thirtieth annual ACM symposium on Theory of computing, 1998.

[5] H. Sun and S. A. Jafar, "The capacity of private information retrieval," IEEE Transactions on Information Theory, vol. 63, no. 7, pp. 40754088, 2017.

[6] _ "The capacity of robust private information retrieval with colluding databases," IEEE Transactions on Information Theory, vol. 64, no. 4, pp. 2361-2370, 2018.

[7] — "The capacity of symmetric private information retrieval," in IEEE Globecom Workshops (GC Wkshps). IEEE, 2016, pp. 1-5.

[8] K. Banawan and S. Ulukus, "The capacity of private information retrieval from coded databases," IEEE Transactions on Information Theory, vol. 64, no. 3, pp. 1945-1956, 2018.

[9] — - "Multi-message private information retrieval: Capacity results and near-optimal schemes," IEEE Transactions on Information Theory, 2018.

[10] Q. Wang and M. Skoglund, "Symmetric private information retrieval for MDS coded distributed storage," in IEEE International Conference on Communications (ICC), 2017, pp. 1-6.

[11] _ _ "Linear symmetric private information retrieval for MDS coded distributed storage with colluding servers," in IEEE Information Theory Workshop (ITW), 2017, pp. 71-75.

[12] N. B. Shah, K. Rashmi, and K. Ramchandran, "One extra bit of download ensures perfectly private information retrieval," in Proc. IEEE Int. Symp. Information Theory, 2014, pp. 856-860.

[13] A. Fazeli, A. Vardy, and E. Yaakobi, "PIR with low storage overhead: coding instead of replication," arXiv preprint arXiv:1505.06241, 2015.

[14] T. H. Chan, S.-W. Ho, and H. Yamamoto, "Private information retrieval for coded storage," in Proc. IEEE Int. Symp. Information Theory, 2015, pp. 2842-2846.

[15] R. Tajeddine and S. E. Rouayheb, "Private information retrieval from MDS coded data in distributed storage systems," in Proc. IEEE Int. Symp. Information Theory, 2016.

[16] R. Freij-Hollanti, O. W. Gnilke, C. Hollanti, and D. A. Karpuk, "Private information retrieval from coded databases with colluding servers," SIAM Journal on Applied Algebra and Geometry, vol. 1, no. 1, pp. 647-664, 2017.

[17] M. Médard and A. Sprintson, Network coding: Fundamentals and applications. Academic Press, 2011.

[18] Q. Wang, H. Sun, and M. Skoglund, "The capacity of private information retrieval with eavesdroppers," arXiv preprint arXiv:1804.10189, 2018.
[19] S. Jaggi, M. Langberg, S. Katti, T. Ho, D. Katabi, and M. Médard, "Resilient network coding in the presence of byzantine adversaries," in 26th IEEE International Conference on Computer Communications (INFOCOM), 2007, pp. 616-624

[20] O. Kosut, L. Tong, and N. David, "Polytope codes against adversaries in networks," IEEE Transactions on Information Theory, vol. 60, no. 6, pp. 3308-3344, 2014

[21] S. Pawar, S. El Rouayheb, and K. Ramchandran, "Securing dynamic distributed storage systems against eavesdropping and adversarial attacks," IEEE Transactions on Information Theory, vol. 57, no. 10, pp. 6734-6753, 2011.

[22] T. M. Cover and J. A. Thomas, Elements of information theory. John Wiley \& Sons, 2012.

[23] H. Yang, W. Shin, and J. Lee, "Private information retrieval for secure distributed storage systems," IEEE Transactions on Information Forensics and Security, vol. 13, no. 12, pp. 2953-2964, 2018.

Qiwen Wang received her B.Sc. degree in Mathematics and B.Eng. degree in Information Engineering in 2010, and Ph.D. degree in Information Engineering in 2015, all from the Chinese University of Hong Kong, Hong Kong. She is currently a postdoctoral researcher in the Department of Information Science and Engineering, KTH Royal Institute of Technology, Stockholm, Sweden.

Mikael Skoglund (S'93-M'97-SM'04) received the Ph.D. degree in 1997 from Chalmers University of Technology, Sweden. In 1997, he joined the Royal Institute of Technology (KTH), Stockholm, Sweden, where he was appointed to the Chair in Communication Theory in 2003. At KTH, he heads the Department of Information Science and Engineering.

Dr. Skoglund has worked on problems in source-channel coding, coding and transmission for wireless communications, Shannon theory, information and control, and statistical signal processing. He has authored and co-authored more than 140 journal and some 340 conference papers.

Dr. Skoglund has served on numerous technical program committees for IEEE sponsored conferences. During 2003-08 he was an associate editor with the IEEE Transactions on Communications and during 2008-12 he was on the editorial board for the IEEE Transactions on Information Theory. He is general co-chair for the upcoming IEEE ITW 2019. 